\title{
A dynamic approach to selecting timepoints for short-term scheduling with application to multipurpose facilities (Supporting Information)
}

\author{
Zachariah Stevenson $^{\dagger}$ Ricardo Fukasawa, $^{\dagger}$ and Luis Ricardez-Sandoval ${ }^{*, \dagger}$ \\ $\dagger$ Dept. of Combinatorics and Optimization, University of Waterloo, Waterloo, ON, \\ N2L 3G1, Canada \\ $\ddagger$ Dept. of Chemical Engineering, University of Waterloo, Waterloo, ON, N2L 3G1, Canada \\ E-mail: laricard@uwaterloo.ca
}

Section S1 presents results and discussion regarding the behaviour of our framework analyzed on a per-iteration basis.

Section S2 presents two tables which contain further information regarding input data for the case study presented in sections 4.3 - 4.5 of the main manuscript. Table S3 includes the normalized process parameter values used for our experiments. The first column is the process index, the second column includes the capacity of each process resource as a portion of the largest resource capacity among all processes. The third presents the processing time of each process as a portion of the greatest processing time over all processes. The fourth column includes the number of resources available for each process. Table S4 presents detailed information about the results that were reported in Section 5 concerning the performance of dynamic timepoint policies. The first column is the instance number of the entry, the second column refers to the amount of elapsed time the entry corresponds to, and the third and fourth columns include the size of the instance when using the static NUD60 discretization. 
The fifth and sixth columns refer to the number of days that were scheduled for the instances and the number of samples that were scheduled. The seventh column displays the size category that the instance was sorted into. The remaining columns indicate the relative performance of each policy at the time of the relevant checkpoint. 


\section{S1 Per Iteration Analysis}

In this section we analyze the behavior of the dynamic framework on the medium sized instances with respect to each iteration. We choose to discuss the medium size instances here because most of the instances tested fell into this category (see Table 3 from the main manuscript); however the observed trends are similar for the small and large sized instances and are not shown for brevity. Note that for these medium sized instances, the maximum number of iterations that were performed by the 60 - 1.05 - UD240 policy was five, and therefore data for this policy does not show up in the results for iterations greater than five. Furthermore, because a given policy will not necessarily perform the same number of iterations on each problem instance (e.g. the 5 - 0.0 - UD120 policy may perform 3 iterations for medium sized instance 1, and perform 8 iterations for medium sized instance 2), the averages reported in this section are obtained using less data points for later iterations than earlier iterations.

The actual number of instances that were used for each policy and iteration number are presented in Table S1. The first column denotes the iteration number, and columns $2-4$ denote how many instances were run for each policy such that the policy used that many iterations. For example, Table S1 shows that there were 28 instances where the 5 - 0.0 UD60 policy took at least 3 iterations to complete, but only 22 instances where it took at least 4 iterations to complete. All policies terminated within 14 iterations over all medium sized instances. From Table S1 we are able to observe that the policies which start with more coarse uniform discretizations generally iterate more times before termination. This is expected as the more coarse policies start with fewer timepoints and therefore should be able to be solved more quickly. Since we impose the same its_tl time limit for each policy, then using a more coarse discretization should allow for more iterations. This does not hold for the 60 - 1.05 - UD240 policy however because of the 5\% improvement that we impose between iterations. We observe that most instances complete in 3 iterations, with all of the instances completing within 5 iterations. 
Table S1: Number of instances considered for each iteration number.

\begin{tabular}{|c|c|c|c|c|}
\hline $\begin{array}{c}\text { Iteration } \\
\text { Number }\end{array}$ & $\begin{array}{c}5-0.0-\text { UD60 } \\
\text { (\# Instances) }\end{array}$ & $\begin{array}{c}5-0.0-\text { UD120 } \\
\text { \# Instances) }\end{array}$ & $\begin{array}{c}5-0.0-\text { UD240 } \\
\text { \# Instances })\end{array}$ & $\begin{array}{c}60-1.0 .5-\text { UD240 } \\
\text { (\# Instances) }\end{array}$ \\
\hline \hline 1 & 34 & 34 & 34 & 34 \\
\hline 2 & 34 & 34 & 34 & 34 \\
\hline 3 & 28 & 33 & 34 & 10 \\
\hline 4 & 22 & 27 & 34 & 2 \\
\hline 5 & 21 & 23 & 31 & \\
\hline 6 & 18 & 23 & 28 & \\
\hline 7 & 13 & 17 & 21 & \\
\hline 8 & 9 & 11 & 15 & \\
\hline 9 & 7 & 9 & 12 & \\
\hline 10 & 4 & 5 & 9 & \\
\hline 11 & 3 & 4 & 4 & \\
\hline 12 & 3 & 4 & 3 & \\
\hline 13 & 3 & 2 & 2 & \\
\hline 14 & 3 & 3 & & \\
\hline
\end{tabular}

We now analyze the average number of timepoints considered for each iteration. These results are presented in Figure S1. Based on the discussion on the average number of timepoint modifications above, the results of Figure $\mathrm{S} 1$ are expected. In the first iteration, we are able to observe the differences in the number of timepoints for the starting discretizations considered, UD60, UD120, and UD240. After the first iteration, we see a moderate increase in the number of timepoints for the 5 - 0.0 - UD120, 5 - 0.0 - UD240, and 60 - 1.05 - UD240 discretizations, while there is a decline in the number of timepoints for policy 5 - 0.0 - UD60, as it was depicted by Figure S2a. These observations support the idea that policies which start with coarse discretizations are able to identify many new timepoints which may be beneficial to a schedule formulation, whereas the policies which start with fine discretizations are not able to identify as many timepoints to add because many are already included. Note that the average number of timepoints considered by the NUD60 static policy for small, medium, and large instance sizes are as follows: small instances - 18,117, medium instances - 45,763, large instances - 92,384. Using these numbers and referring again to Figure S1 we observe more than a $75 \%$ reduction in the number of timepoints considered in some cases 
by using a dynamic policy. This reduction in timepoints also translates into a reduction in problem size as shown in Table S2. This table presents the average problem size (in terms of number of variables and constraints) for each policy and iteration and compares it to the average problem size of the NUD60 discretization. The maximum problem sizes among all iterations for each policy are in bold. Therefore, reducing the number of timepoints may be useful in cases where the size of the problems considered surpasses the capabilities of the available hardware (e.g. CPU or memory limitations). Instead of using a fine static discretization, a user may iteratively refine the time grids by solving smaller sized problems, yet still achieve good solution quality as discussed in the earlier results sections $4.3-4.5$ from the main manuscript.

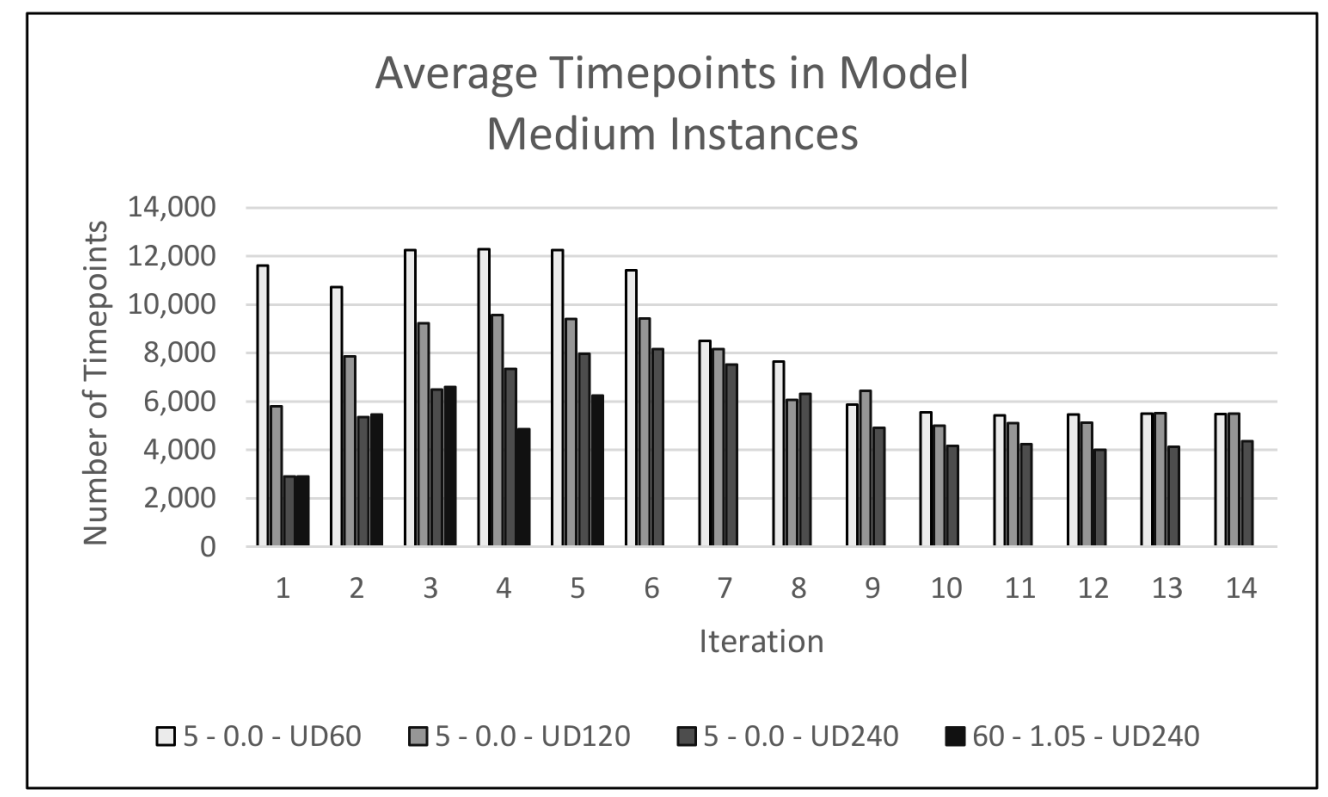

Figure S1: Per iteration timepoint differences for medium sized instances.

We now discuss the average number of timepoints that were added, removed, and their difference between iterations. This information is presented by Figure S2. We may immediately discern that regardless of the policy considered, after a few iterations there is a sharp decline in both the number of timepoints that are being added and the number of timepoints that are being removed between iterations. This trend shows that the timepoint sets undergo large changes at the beginning of the procedure and then become relatively stable after a 
few iterations. Moreover, we observe that there are a large number of timepoints which are removed in the initial iterations. The number of timepoints removed is directly related to the granularity of the initial discretization, where more fine discretizations have more timepoints removed and in particular the 5-0.0 - UD60 policy removes more timepoints than it adds after the first iteration. This shows that using a uniform discretization will include many timepoints which are not required for obtaining high quality solutions. By observing the relative magnitudes of the timepoint changes between iterations, we observe that the amount of timepoints modifications done by policies which start with more coarse discretizations decreases more slowly than policies which start with more fine discretizations. This is reasonable because the quality of the initial solutions is directly related to the granularity of the starting discretization. Starting with a fine discretization allows us to make many timepoint modifications initially but then quickly converge to a suitable set of timepoints.

Figure S3a presents the per iteration performance improvements for each policy, in terms of objective value percentage increase. Similar to the results discussed above when considering timepoint differences between iterations, most of the performance improvements are obtained within the first few iterations of the framework. This is unsurprising as we have already noted that the timepoint sets are only marginally changed in the later iterations so we do not expect to gain as much improvement then. These results suggest that only a few iterations are necessary and that perhaps a stopping criteria based on the number of iterations carried out may allow us to obtain most of the benefits of the procedure, without incurring all of the computational cost.

Figure S3b shows the time taken to complete each iteration for each policy. From this figure, we observe an initial increase in the solving time per iteration. This increase corresponds to the rapidly changing timepoint sets which render previously obtained solutions not as relevant a starting solution as for later iterations where timepoints do not change much. After the initial period of increasing solution times, we see that the time per iteration begins to decrease and stabilize to approximately 50 seconds. By limiting the number of iterations 


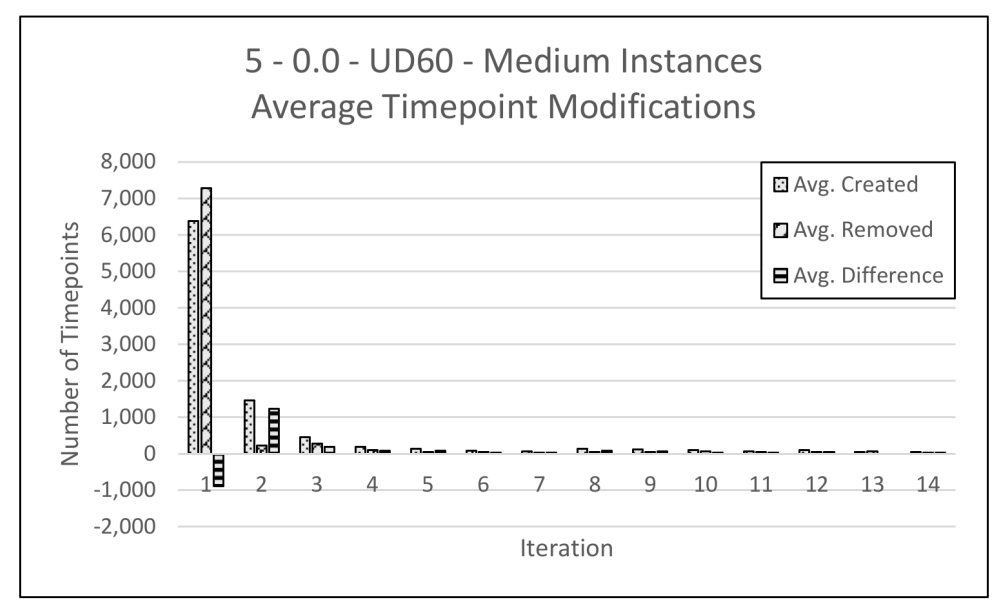

(a) Timepoint modifications for 5 - 0.0 - UD60 policy.

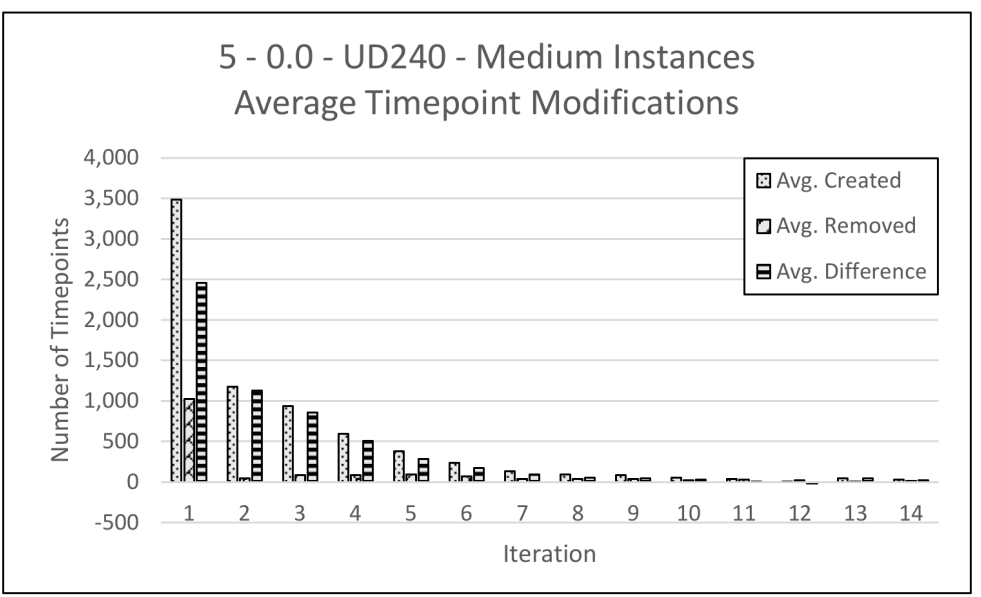

(c) Timepoint modifications for 5 - 0.0 - UD240 policy.

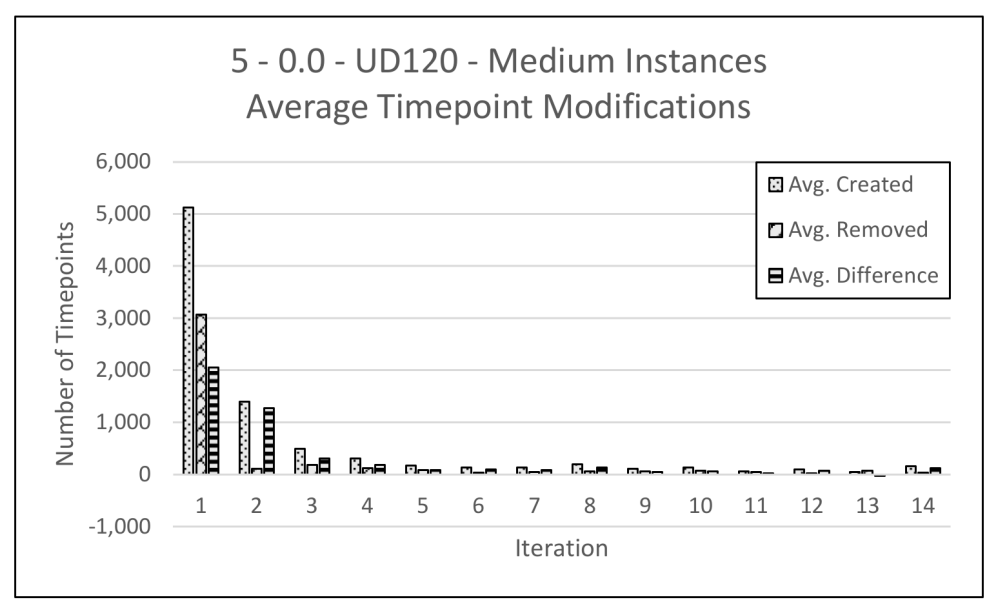

(b) Timepoint modifications for 5 - 0.0 - UD120 policy.

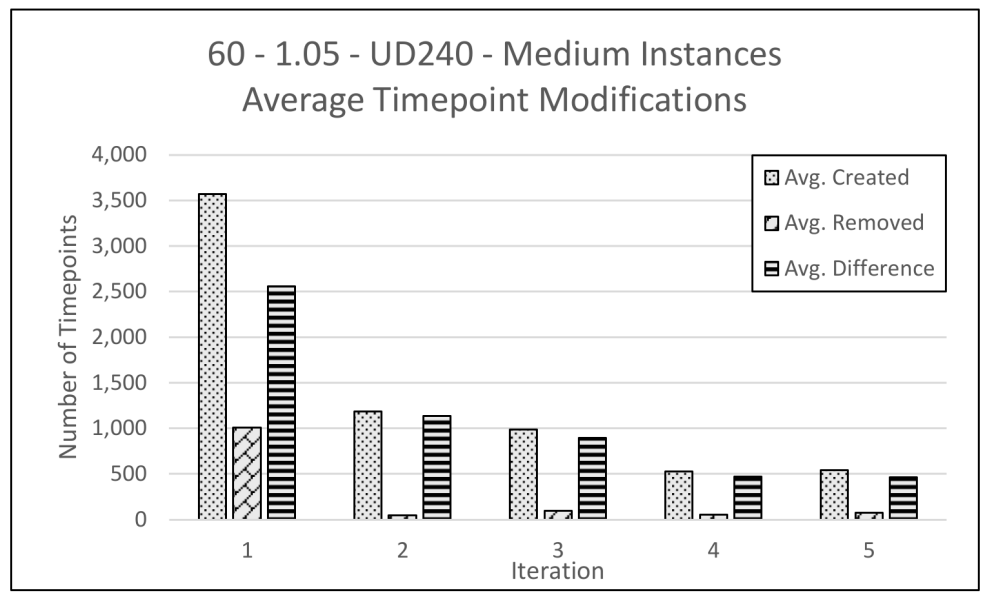

(d) Timepoint modifications for 60 - 1.05 - UD240 policy.

Figure S2: The average number of timepoints added, removed, and their difference between iterations over each policy. 
as suggested previously, we may remove the extra time spent in the later iterations without much improvement.

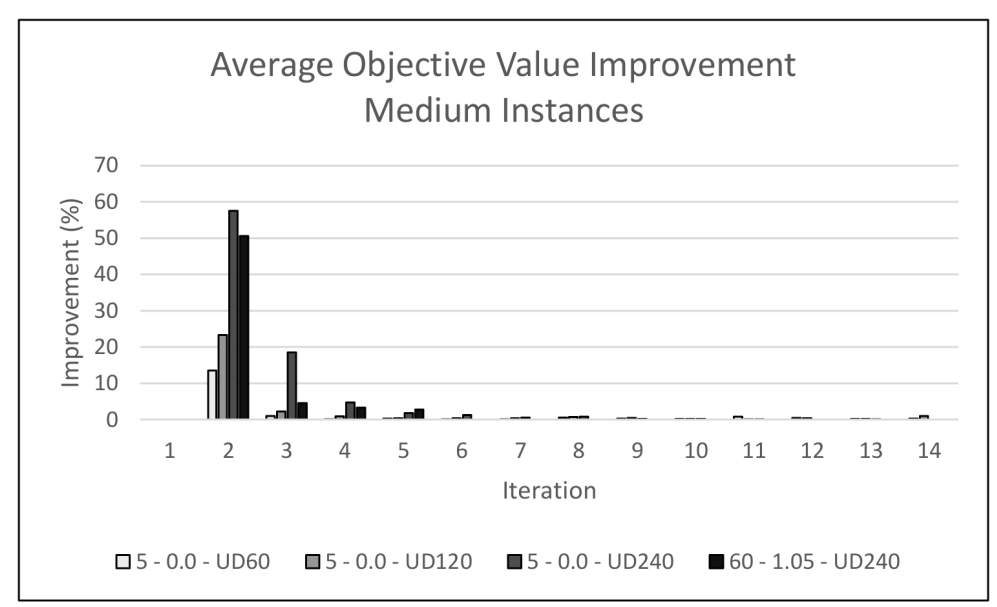

(a) Per iteration objective value improvements.

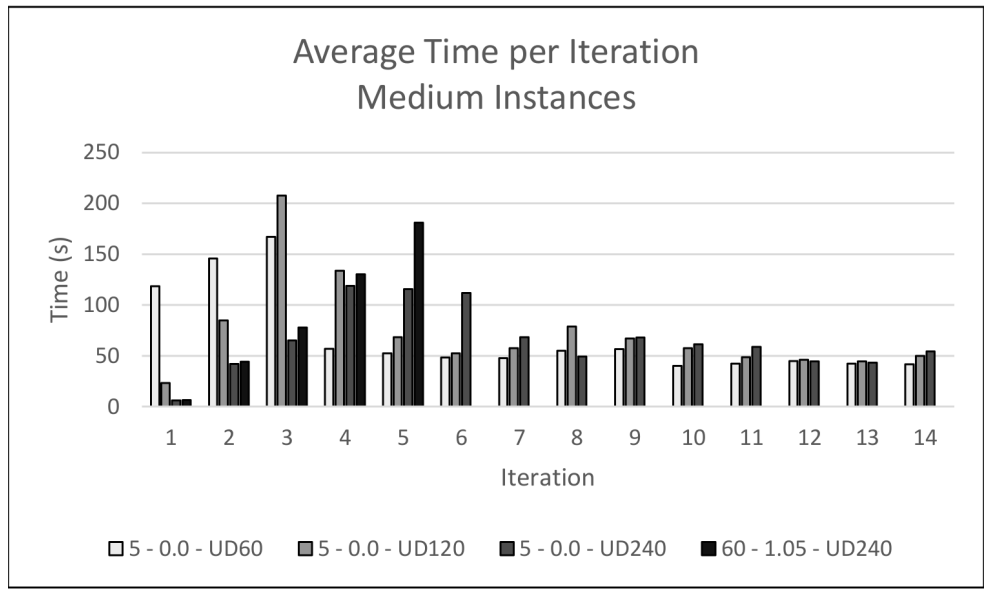

(b) Time taken per iteration.

Figure S3: Average objective value improvements and time taken per iteration.

Table S2: Average number of variables and constraints per iteration, numbers reported in 1,000 's.

\begin{tabular}{|c|c|c|c|c|c|c|c|c|c|c|}
\hline \multirow{2}{*}{$\begin{array}{l}\text { Iteration } \\
\text { Number }\end{array}$} & \multicolumn{2}{|c|}{$5-0.0-$ UD60 } & \multicolumn{2}{|c|}{$5-0.0-\mathrm{UD} 120$} & \multicolumn{2}{|c|}{$5-0.0-\mathrm{UD} 240$} & \multicolumn{2}{|c|}{60 - 1.05 - UD240 } & \multicolumn{2}{|c|}{ NUD60 } \\
\hline & Vars & Cons & Vars & Cons & Vars & Cons & Vars & Cons & Vars & Cons \\
\hline 1 & 329 & 192 & 171 & 101 & 93 & 56 & 93 & 56 & \multirow{14}{*}{1,611} & \multirow{14}{*}{900} \\
\hline 2 & 1,191 & 621 & 1,002 & 521 & 796 & 412 & 802 & 416 & & \\
\hline 3 & 1,250 & 654 & 1,121 & 583 & 928 & 481 & 935 & 485 & & \\
\hline 4 & 1,271 & 664 & 1,122 & 584 & 1,034 & 536 & 1,108 & 569 & & \\
\hline 5 & 1,268 & 663 & 1,129 & 588 & 1,088 & 564 & 951 & 493 & & \\
\hline 6 & 1,304 & 680 & 1,156 & 601 & 1,106 & 573 & $\mathrm{~N} / \mathrm{A}$ & $\mathrm{N} / \mathrm{A}$ & & \\
\hline 7 & 1,348 & 696 & 1,213 & 628 & 1,086 & 562 & $\mathrm{~N} / \mathrm{A}$ & $\mathrm{N} / \mathrm{A}$ & & \\
\hline 8 & 1.428 & 735 & 1,431 & 734 & 1,170 & 603 & $\mathrm{~N} / \mathrm{A}$ & $\mathrm{N} / \mathrm{A}$ & & \\
\hline 9 & 1,442 & 739 & 1,369 & 704 & 1,300 & 666 & $\mathrm{~N} / \mathrm{A}$ & $\mathrm{N} / \mathrm{A}$ & & \\
\hline 10 & 1,156 & 595 & 1,165 & 598 & 1,396 & 713 & $\mathrm{~N} / \mathrm{A}$ & $\mathrm{N} / \mathrm{A}$ & & \\
\hline 12 & 1,179 & 606 & 1,131 & 581 & 1,114 & 570 & $\mathrm{~N} / \mathrm{A}$ & $\mathrm{N} / \mathrm{A}$ & & \\
\hline 11 & 1,176 & 604 & 1,133 & 582 & 1,387 & 709 & $\mathrm{~N} / \mathrm{A}$ & $\mathrm{N} / \mathrm{A}$ & & \\
\hline 13 & 1,182 & 608 & 1,176 & 605 & 1,133 & 580 & $\mathrm{~N} / \mathrm{A}$ & $\mathrm{N} / \mathrm{A}$ & & \\
\hline 14 & 1,175 & 604 & 1,141 & 587 & 1,109 & 569 & $\mathrm{~N} / \mathrm{A}$ & $\mathrm{N} / \mathrm{A}$ & & \\
\hline
\end{tabular}




\section{S2 Additional Case Study Data}

Table S3: Normalized process parameters used during experiments.

\begin{tabular}{|c|c|c|c|}
\hline $\begin{array}{c}\text { Process ID } \\
\qquad(u)\end{array}$ & $\begin{array}{l}\text { Process } \\
\text { Capacity } \\
(\kappa(u))\end{array}$ & $\begin{array}{l}\text { Processing } \\
\text { Time }(\tau(u))\end{array}$ & $\begin{array}{c}\text { Number of } \\
\text { Resources } \\
(\rho(u))\end{array}$ \\
\hline 1 & 0.724638 & 0.001488 & 2 \\
\hline 2 & 0.043478 & 0.005952 & 3 \\
\hline 3 & 0.039855 & 0.047619 & 1 \\
\hline 4 & 0.000725 & 0.142857 & 4500 \\
\hline 5 & 0.362319 & 0.037202 & 4 \\
\hline 6 & 0.036232 & 0.018651 & 10 \\
\hline 7 & 0.036232 & 0.099206 & 2 \\
\hline 8 & 0.036232 & 0.018651 & 6 \\
\hline 9 & 0.036232 & 0.019841 & 2 \\
\hline 10 & 0.002174 & 0.000794 & 1 \\
\hline 11 & 0.036232 & 0.019841 & 3 \\
\hline 12 & 0.036232 & 0.014881 & 1 \\
\hline 13 & 0.036232 & 0.00496 & 3 \\
\hline 14 & 0.07971 & 0.107143 & 1 \\
\hline 15 & 0.036232 & 0.009921 & 1 \\
\hline 16 & 0.030435 & 0.003968 & 2 \\
\hline 17 & 0.030435 & 0.003968 & 2 \\
\hline 18 & 0.021739 & 0.10119 & 4 \\
\hline 19 & 0.156522 & 0.029762 & 2 \\
\hline 20 & 0.015217 & 0.014881 & 2 \\
\hline
\end{tabular}

Continued on next page 
Table S3 - Continued from previous page

\begin{tabular}{|c|c|c|c|}
\hline $\begin{array}{c}\text { Process ID } \\
\qquad(u)\end{array}$ & $\begin{array}{l}\text { Process } \\
\text { Capacity } \\
(\kappa(u))\end{array}$ & $\begin{array}{c}\text { Processing } \\
\text { Time }(\tau(u))\end{array}$ & $\begin{array}{c}\text { Number of } \\
\text { Resources } \\
(\rho(u))\end{array}$ \\
\hline 21 & 0.005797 & 0.018849 & 1 \\
\hline 22 & 0.034783 & 0.061012 & 1 \\
\hline 23 & 0.000725 & 0.016567 & 1 \\
\hline 24 & 0.005072 & 0.142857 & 1 \\
\hline 25 & 0.042754 & 0.209425 & 1 \\
\hline 26 & 0.028986 & 0.165179 & 2 \\
\hline 27 & 0.028986 & 0.744048 & 40 \\
\hline 28 & 0.021739 & 0.035714 & 2 \\
\hline 29 & 0.005797 & 0.053571 & 1 \\
\hline 30 & 0.03913 & 0.050595 & 4 \\
\hline 31 & 0.021739 & 0.005952 & 1 \\
\hline 32 & 0.019565 & 0.011409 & 1 \\
\hline 33 & 0.019565 & 0.040675 & 1 \\
\hline 34 & 0.018841 & 0.013889 & 1 \\
\hline 35 & 0.018841 & 0.013889 & 1 \\
\hline 36 & 0.018841 & 0.013889 & 1 \\
\hline 37 & 0.018116 & 0.0125 & 1 \\
\hline 38 & 0.017391 & 0.013889 & 1 \\
\hline 39 & 0.019565 & 0.010913 & 1 \\
\hline 40 & 0.144928 & 0.011905 & 1 \\
\hline 41 & 0.073913 & 0.011905 & 2 \\
\hline
\end{tabular}

Continued on next page 
Table S3 - Continued from previous page

\begin{tabular}{|c|c|c|c|}
\hline $\begin{array}{c}\text { Process ID } \\
(u)\end{array}$ & $\begin{array}{c}\text { Process } \\
\text { Capacity } \\
(\kappa(u))\end{array}$ & $\begin{array}{l}\text { Processing } \\
\text { Time }(\tau(u))\end{array}$ & $\begin{array}{c}\text { Number of } \\
\text { Resources } \\
(\rho(u))\end{array}$ \\
\hline 42 & 0.05942 & 0.000992 & 2 \\
\hline 43 & 0.221014 & 0.011905 & 2 \\
\hline 44 & 0.108696 & 0.02381 & 1 \\
\hline 45 & 0.074638 & 0.005952 & 1 \\
\hline 46 & 0.09058 & 0.017857 & 1 \\
\hline 47 & 0.09058 & 0.017857 & 1 \\
\hline 48 & 0.008696 & 0.005952 & 1 \\
\hline 49 & 0.042754 & 0.008929 & 1 \\
\hline 50 & 0.014493 & 0.001984 & 1 \\
\hline 51 & 0.043478 & 0.005952 & 1 \\
\hline 52 & 0.004348 & 0.005952 & 2 \\
\hline 53 & 0.028986 & 0.005952 & 1 \\
\hline 54 & 0.043478 & 0.005952 & 1 \\
\hline 55 & 0.021739 & 0.005952 & 2 \\
\hline 56 & 0.347826 & 0.017857 & 3 \\
\hline 57 & 0.318841 & 0.02381 & 2 \\
\hline 58 & 0.072464 & 0.041667 & 1 \\
\hline 59 & 0.036232 & 0.098214 & 1 \\
\hline 60 & 0.008696 & 0.013393 & 2 \\
\hline 61 & 0.021739 & 0.002976 & 1 \\
\hline 62 & 0.005797 & 0.026786 & 3 \\
\hline
\end{tabular}

Continued on next page 
Table S3 - Continued from previous page

\begin{tabular}{|c|c|c|c|}
\hline $\begin{array}{c}\text { Process ID } \\
\qquad(u)\end{array}$ & $\begin{array}{l}\text { Process } \\
\text { Capacity } \\
(\kappa(u))\end{array}$ & $\begin{array}{c}\text { Processing } \\
\text { Time }(\tau(u))\end{array}$ & $\begin{array}{c}\text { Number of } \\
\text { Resources } \\
(\rho(u))\end{array}$ \\
\hline 63 & 0.156522 & 0.011905 & 4 \\
\hline 64 & 0.313043 & 0.021825 & 2 \\
\hline 65 & 0.318841 & 0.021825 & 4 \\
\hline 66 & 0.021739 & 0.005952 & 5 \\
\hline 67 & 0.052174 & 0.014385 & 1 \\
\hline 68 & 0.049275 & 0.02629 & 1 \\
\hline 69 & 0.037681 & 0.048611 & 1 \\
\hline 70 & 0.021739 & 0.066964 & 1 \\
\hline 71 & 0.000725 & 0.024306 & 2 \\
\hline 72 & 0.013043 & 0.048115 & 1 \\
\hline 73 & 0.001449 & 0.001984 & 2 \\
\hline 74 & 0.043478 & 0.005952 & 1 \\
\hline 75 & 0.030435 & 0.006448 & 2 \\
\hline 76 & 0.030435 & 0.000992 & 2 \\
\hline 77 & 0.06087 & 0.005952 & 1 \\
\hline 78 & 0.004348 & 0.032738 & 2 \\
\hline 79 & 0.030435 & 0.004167 & 2 \\
\hline 80 & 0.013043 & 0.001488 & 2 \\
\hline 81 & 0.030435 & 0.001488 & 2 \\
\hline 82 & 0.030435 & 0.007937 & 2 \\
\hline 83 & 0.013043 & 0.007937 & 2 \\
\hline
\end{tabular}

Continued on next page 
Table S3 - Continued from previous page

\begin{tabular}{|c|c|c|c|}
\hline $\begin{array}{c}\text { Process ID } \\
\qquad(u)\end{array}$ & $\begin{array}{c}\text { Process } \\
\text { Capacity } \\
(\kappa(u))\end{array}$ & $\begin{array}{c}\text { Processing } \\
\text { Time }(\tau(u))\end{array}$ & $\begin{array}{c}\text { Number of } \\
\text { Resources } \\
(\rho(u))\end{array}$ \\
\hline 84 & 0.030435 & 0.004167 & 1 \\
\hline 85 & 0.030435 & 0.004167 & 2 \\
\hline 86 & 0.010145 & 0.047619 & 1 \\
\hline 87 & 0.021739 & 0.006746 & 1 \\
\hline 88 & 0.092754 & 0.073016 & 4 \\
\hline 89 & 0.092754 & 0.006349 & 2 \\
\hline 90 & 0.000725 & 0.000992 & 1 \\
\hline 91 & 0.000725 & 0.142857 & 1 \\
\hline 92 & 0.108696 & 0.142857 & 2 \\
\hline 93 & 0.018841 & 0.012897 & 1 \\
\hline 94 & 0.018841 & 0.012897 & 1 \\
\hline 95 & 0.018116 & 0.013988 & 1 \\
\hline 96 & 0.018841 & 0.011905 & 1 \\
\hline 97 & 0.019565 & 0.042659 & 1 \\
\hline 98 & 0.130435 & 0.03869 & 1 \\
\hline 99 & 0.173913 & 0.142857 & 1 \\
\hline 100 & 0.082609 & 0.31498 & 1 \\
\hline 101 & 0.084058 & 0.01746 & 1 \\
\hline 102 & 0.521739 & 0.072917 & 10 \\
\hline 103 & 0.347826 & 0.046726 & 10 \\
\hline 104 & 0.521739 & 0.070536 & 10 \\
\hline
\end{tabular}

Continued on next page 
Table S3 - Continued from previous page

\begin{tabular}{|c|c|c|c|}
\hline $\begin{array}{c}\text { Process ID } \\
(u)\end{array}$ & $\begin{array}{c}\text { Process } \\
\text { Capacity } \\
(\kappa(u))\end{array}$ & $\begin{array}{l}\text { Processing } \\
\text { Time }(\tau(u))\end{array}$ & $\begin{array}{c}\text { Number of } \\
\text { Resources } \\
(\rho(u))\end{array}$ \\
\hline 105 & 0.121739 & 0.017857 & 10 \\
\hline 106 & 0.217391 & 0.024802 & 10 \\
\hline 107 & 1.0 & 0.138393 & 10 \\
\hline 108 & 0.26087 & 0.025595 & 2 \\
\hline 109 & 0.081159 & 0.124603 & 8 \\
\hline 110 & 0.097826 & 0.113194 & 8 \\
\hline 111 & 0.05942 & 0.025198 & 8 \\
\hline 112 & 0.05942 & 0.142659 & 8 \\
\hline 113 & 0.053623 & 0.127083 & 8 \\
\hline 114 & 0.374638 & 0.159226 & 8 \\
\hline 115 & 0.195652 & 0.077976 & 8 \\
\hline 116 & 0.191304 & 0.133929 & 1 \\
\hline 117 & 0.15 & 0.136905 & 5 \\
\hline 118 & 0.007971 & 0.000298 & 6 \\
\hline 119 & 0.021739 & 0.000992 & 1 \\
\hline 120 & 0.021739 & 0.000992 & 1 \\
\hline 121 & 0.007971 & 0.002976 & 1 \\
\hline 122 & 0.007971 & 0.000694 & 1 \\
\hline 123 & 0.347826 & 0.047619 & 1 \\
\hline 124 & 0.386232 & 0.047619 & 1 \\
\hline 125 & 0.104348 & 0.047619 & 1 \\
\hline
\end{tabular}

Continued on next page 
Table S3 - Continued from previous page

\begin{tabular}{|c|c|c|c|}
\hline $\begin{array}{c}\text { Process ID } \\
\qquad(u)\end{array}$ & $\begin{array}{l}\text { Process } \\
\text { Capacity } \\
(\kappa(u))\end{array}$ & $\begin{array}{c}\text { Processing } \\
\text { Time }(\tau(u))\end{array}$ & $\begin{array}{c}\text { Number of } \\
\text { Resources } \\
(\rho(u))\end{array}$ \\
\hline 126 & 0.26087 & 1.0 & 1 \\
\hline 127 & 0.521739 & 1.0 & 1 \\
\hline 128 & 0.072464 & 0.041667 & 1 \\
\hline 129 & 0.065217 & 0.029762 & 2 \\
\hline 130 & 0.03913 & 0.012897 & 1 \\
\hline 131 & 0.008696 & 0.125 & 1 \\
\hline 132 & 0.03913 & 0.102183 & 1 \\
\hline 133 & 0.03913 & 0.012897 & 1 \\
\hline 134 & 0.011594 & 0.02629 & 1 \\
\hline 135 & 0.011594 & 0.029762 & 1 \\
\hline 136 & 0.03913 & 0.019841 & 1 \\
\hline 137 & 0.018841 & 0.075496 & 1 \\
\hline 138 & 0.03913 & 0.007937 & 2 \\
\hline 139 & 0.018841 & 0.030556 & 1 \\
\hline 140 & 0.03913 & 0.008929 & 4 \\
\hline 141 & 0.069565 & 0.064782 & 3 \\
\hline 142 & 0.069565 & 0.006944 & 4 \\
\hline 143 & 0.104348 & 0.020536 & 1 \\
\hline 144 & 0.073913 & 0.091071 & 1 \\
\hline 145 & 0.073913 & 0.040476 & 1 \\
\hline 146 & 0.073913 & 0.040476 & 1 \\
\hline
\end{tabular}

Continued on next page 
Table S3 - Continued from previous page

\begin{tabular}{|c|c|c|c|}
\hline $\begin{array}{c}\text { Process ID } \\
\qquad(u)\end{array}$ & $\begin{array}{l}\text { Process } \\
\text { Capacity } \\
(\kappa(u))\end{array}$ & $\begin{array}{c}\text { Processing } \\
\text { Time }(\tau(u))\end{array}$ & $\begin{array}{c}\text { Number of } \\
\text { Resources } \\
(\rho(u))\end{array}$ \\
\hline 147 & 0.043478 & 0.005952 & 1 \\
\hline 148 & 0.010145 & 0.166667 & 2 \\
\hline 149 & 0.010145 & 0.044643 & 2 \\
\hline 150 & 0.005797 & 0.041667 & 2 \\
\hline 151 & 0.005797 & 0.018353 & 1 \\
\hline 152 & 0.005797 & 0.001488 & 2 \\
\hline 153 & 0.043478 & 0.002976 & 2 \\
\hline 154 & 0.015942 & 0.00248 & 2 \\
\hline 155 & 0.005797 & 0.003175 & 1 \\
\hline 156 & 0.000725 & 0.000198 & 1 \\
\hline 157 & 0.013043 & 0.029762 & 1 \\
\hline 158 & 0.019565 & 0.113095 & 1 \\
\hline 159 & 0.015942 & 0.005952 & 1 \\
\hline 160 & 0.014493 & 0.089286 & 1 \\
\hline 161 & 0.000725 & 0.001587 & 1 \\
\hline 162 & 0.000725 & $9.9 \mathrm{e}-5$ & 1 \\
\hline 163 & 0.043478 & 0.105655 & 1 \\
\hline 164 & 0.043478 & 0.105655 & 1 \\
\hline 165 & 0.043478 & 0.105655 & 1 \\
\hline 166 & 0.318841 & 0.160714 & 4 \\
\hline 167 & 0.318841 & 0.166667 & 4 \\
\hline
\end{tabular}

Continued on next page 
Table S3 - Continued from previous page

\begin{tabular}{|c|c|c|c|}
\hline $\begin{array}{c}\text { Process ID } \\
\qquad(u)\end{array}$ & $\begin{array}{l}\text { Process } \\
\text { Capacity } \\
(\kappa(u))\end{array}$ & $\begin{array}{c}\text { Processing } \\
\text { Time }(\tau(u))\end{array}$ & $\begin{array}{c}\text { Number of } \\
\text { Resources } \\
(\rho(u))\end{array}$ \\
\hline 168 & 0.017391 & 0.056548 & 1 \\
\hline 169 & 0.011594 & 0.15873 & 1 \\
\hline 170 & 0.017391 & 0.02381 & 1 \\
\hline 171 & 0.004348 & 0.03869 & 1 \\
\hline 172 & 0.014493 & 0.006151 & 1 \\
\hline 173 & 0.008696 & 0.012401 & 1 \\
\hline 174 & 0.023188 & 0.008929 & 1 \\
\hline 175 & 0.000725 & $9.9 \mathrm{e}-5$ & 6 \\
\hline 176 & 0.000725 & $9.9 \mathrm{e}-5$ & 1 \\
\hline 177 & 0.01087 & 0.001488 & 1 \\
\hline 178 & 0.000725 & $9.9 \mathrm{e}-5$ & 1 \\
\hline 179 & 0.000725 & 0.000893 & 1 \\
\hline 180 & 0.000725 & $9.9 \mathrm{e}-5$ & 1 \\
\hline 181 & 0.000725 & 0.000992 & 3 \\
\hline 182 & 0.008696 & 0.016667 & 1 \\
\hline 183 & 0.000725 & 0.001488 & 1 \\
\hline 184 & 0.000725 & $9.9 \mathrm{e}-5$ & 1 \\
\hline 185 & 0.004348 & 0.002183 & 5 \\
\hline 186 & 0.000725 & $9.9 \mathrm{e}-5$ & 2 \\
\hline 187 & 0.724638 & 0.000992 & 1 \\
\hline 188 & 0.724638 & 0.142857 & 1 \\
\hline 189 & 0.724638 & 0.142857 & 1 \\
\hline
\end{tabular}


Table S4: Results for each instance of dynamic timepoint study.

\begin{tabular}{|c|c|c|c|c|c|c|c|c|c|c|c|c|c|c|}
\hline 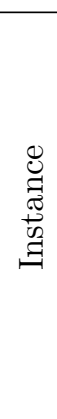 & 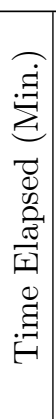 & 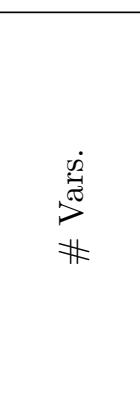 & $\begin{array}{l}\dot{0} \\
\dot{0} \\
\text { \# } \\
\text { \# }\end{array}$ & 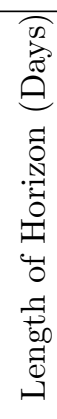 & 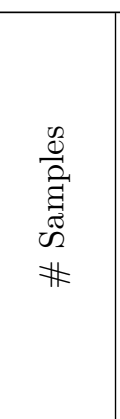 & $\stackrel{\stackrel{N}{N}}{i n}$ & $\begin{array}{l}\stackrel{8}{\circ} \\
\text { Z }\end{array}$ & 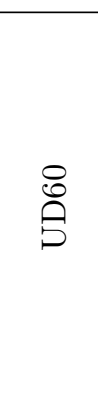 & $\frac{\stackrel{\curvearrowright}{\sim}}{\vec{\rho}}$ & $\begin{array}{l}\stackrel{ }{N} \\
\text { S } \\
\text { S }\end{array}$ & $\begin{array}{l}8 \\
\stackrel{0}{S} \\
\stackrel{5}{1} \\
1 \\
1 \\
10\end{array}$ & 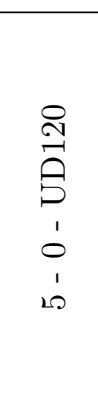 & $\begin{array}{l}\text { P } \\
\text { N } \\
\text { م } \\
1 \\
0 \\
1 \\
10\end{array}$ & 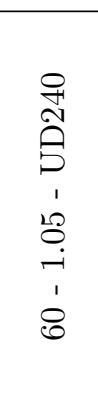 \\
\hline 1 & 1 & 698,866 & 388,218 & 1 & 10,000 & Small & 0.954 & 0.954 & 0.954 & 0.851 & 0.851 & 0.851 & 0.682 & 0.682 \\
\hline 1 & 5 & 698,866 & 388,218 & 1 & 10,000 & Small & 0.954 & 0.954 & 0.851 & 0.851 & 0.682 & 0.682 & 0.486 & 0.486 \\
\hline 1 & 15 & 698,866 & 388,218 & 1 & 10,000 & Small & 0.954 & 0.851 & 0.851 & 0.682 & 0.486 & 0.486 & 0.975 & 0.948 \\
\hline 1 & 30 & 698,866 & 388,218 & 1 & 10,000 & Small & 0.954 & 0.851 & 0.682 & 0.486 & 0 & 0.975 & 0.984 & 1 \\
\hline 1 & 60 & 698,866 & 388,218 & 1 & 10,000 & Small & 0.954 & 0.851 & 0.682 & 0.486 & 0.975 & 0.984 & 1 & 0.973 \\
\hline 2 & 1 & 686,782 & 382,158 & 1 & 10,000 & Small & 0.933 & 0.933 & 0.933 & 0.841 & 0.841 & 0.841 & 0.667 & 0.667 \\
\hline 2 & 5 & 686,782 & 382,158 & 1 & 10,000 & Small & 0.933 & 0.933 & 0.841 & 0.841 & 0.667 & 0.667 & 0.474 & 0.474 \\
\hline 2 & 15 & 686,782 & 382,158 & 1 & 10,000 & Small & 0.933 & 0.841 & 0.841 & 0.667 & 0.474 & 0.474 & 1 & 0.922 \\
\hline 2 & 30 & 686,782 & 382,158 & 1 & 10,000 & Small & 0.933 & 0.841 & 0.667 & 0.474 & 0 & 1 & 0.991 & 0.993 \\
\hline 2 & 60 & 686,782 & 382,158 & 1 & 10,000 & Small & 0.933 & 0.841 & 0.667 & 0.474 & 1 & 0.991 & 0.993 & 0.98 \\
\hline 3 & 1 & 657,822 & 367,693 & 1 & 10,000 & Small & 0.953 & 0.953 & 0.953 & 0.842 & 0.842 & 0.842 & 0.658 & 0.658 \\
\hline 3 & 5 & 657,822 & 367,693 & 1 & 10,000 & Small & 0.953 & 0.953 & 0.842 & 0.842 & 0.658 & 0.658 & 0.461 & 0.461 \\
\hline 3 & 15 & 657,822 & 367,693 & 1 & 10,000 & Small & 0.953 & 0.842 & 0.842 & 0.658 & 0.461 & 0.461 & 0.98 & 0.924 \\
\hline 3 & 30 & 657,822 & 367,693 & 1 & 10,000 & Small & 0.953 & 0.842 & 0.658 & 0.461 & 0 & 0.98 & 0.976 & 1 \\
\hline 3 & 60 & 657,822 & 367,693 & 1 & 10,000 & Small & 0.953 & 0.842 & 0.658 & 0.461 & 0.98 & 0.976 & 1 & 0.998 \\
\hline 4 & 1 & 646,562 & 361,874 & 1 & 10,000 & Small & 0.973 & 0.973 & 0.973 & 0.864 & 0.864 & 0.864 & 0.684 & 0.684 \\
\hline 4 & 5 & 646,562 & 361,874 & 1 & 10,000 & Small & 0.973 & 0.973 & 0.864 & 0.864 & 0.684 & 0.684 & 0.487 & 0.487 \\
\hline 4 & 15 & 646,562 & 361,874 & 1 & 10,000 & Small & 0.973 & 0.864 & 0.864 & 0.684 & 0.487 & 0.487 & 0.985 & 0.939 \\
\hline 4 & 30 & 646,562 & 361,874 & 1 & 10,000 & Small & 0.973 & 0.864 & 0.684 & 0.487 & 0 & 0.985 & 0.984 & 0.999 \\
\hline 4 & 60 & 646,562 & 361,874 & 1 & 10,000 & Small & 0.973 & 0.864 & 0.684 & 0.487 & 0.985 & 0.984 & 0.999 & 1 \\
\hline 5 & 1 & 662,390 & 369,871 & 1 & 10,000 & Small & 0.956 & 0.956 & 0.956 & 0.839 & 0.839 & 0.839 & 0.669 & 0.669 \\
\hline 5 & 5 & 662,390 & 369,871 & 1 & 10,000 & Small & 0.956 & 0.956 & 0.839 & 0.839 & 0.669 & 0.669 & 0.469 & 0.469 \\
\hline 5 & 15 & 662,390 & 369,871 & 1 & 10,000 & Small & 0.956 & 0.839 & 0.839 & 0.669 & 0.469 & 0.469 & 0.974 & 0.946 \\
\hline 5 & 30 & 662,390 & 369,871 & 1 & 10,000 & Small & 0.956 & 0.839 & 0.669 & 0.469 & 0 & 0.974 & 0.987 & 1 \\
\hline
\end{tabular}


Table S4 - Continued from previous page

\begin{tabular}{|c|c|c|c|c|c|c|c|c|c|c|c|c|c|c|}
\hline 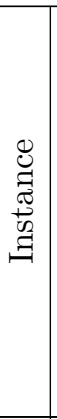 & 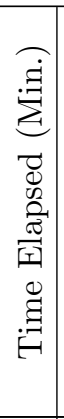 & 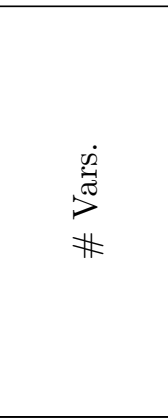 & $\begin{array}{l}\dot{\Xi} \\
\dot{0} \\
0 \\
\#\end{array}$ & 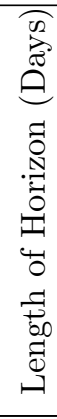 & 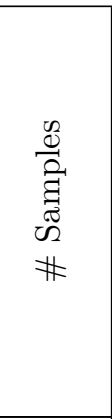 & 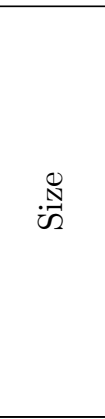 & $\frac{8}{\circ}$ & 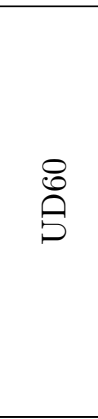 & $\frac{\stackrel{\curvearrowright}{N}}{\vec{\rho}}$ & 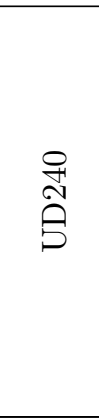 & $\begin{array}{l}8 \\
\stackrel{0}{0} \\
\vdots \\
1 \\
1 \\
1 \\
1\end{array}$ & $\begin{array}{l}\stackrel{\curvearrowright}{N} \\
\vec{D} \\
\vdots \\
1 \\
0 \\
1 \\
10\end{array}$ & $\begin{array}{l}\text { N } \\
\stackrel{N}{N} \\
\text { S } \\
1 \\
0 \\
1 \\
1\end{array}$ & 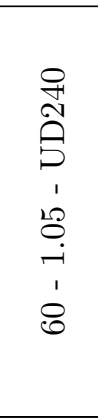 \\
\hline 5 & 60 & 662,390 & 369,871 & 1 & 10,000 & Small & 0.956 & 0.839 & 0.669 & 0.469 & 0.974 & 0.987 & 1 & 0.975 \\
\hline 6 & 1 & $1,245,832$ & 664,038 & 1 & 20,000 & Med. & 0.955 & 0.955 & 0.955 & 0.832 & 0.832 & 0.832 & 0.68 & 0.68 \\
\hline 6 & 5 & $1,245,832$ & 664,038 & 1 & 20,000 & Med. & 0.955 & 0.955 & 0.832 & 0.832 & 0.68 & 0.68 & 0.525 & 0.525 \\
\hline 6 & 15 & $1,245,832$ & 664,038 & 1 & 20,000 & Med. & 0.955 & 0.832 & 0.832 & 0.68 & 0.525 & 0.525 & 0.967 & 0.807 \\
\hline 6 & 30 & $1,245,832$ & 664,038 & 1 & 20,000 & Med. & 0.955 & 0.832 & 0.68 & 0.525 & 1 & 0.967 & 0.994 & 1 \\
\hline 6 & 60 & $1,245,832$ & 664,038 & 1 & 20,000 & Med. & 0.955 & 0.832 & 0.68 & 0.525 & 0.967 & 0.994 & 1 & 0.998 \\
\hline 7 & 1 & $1,318,834$ & 700,531 & 1 & 20,000 & Med. & 0.932 & 0.932 & 0.932 & 0.808 & 0.808 & 0.808 & 0.651 & 0.651 \\
\hline 7 & 5 & $1,318,834$ & 700,531 & 1 & 20,000 & Med. & 0.932 & 0.932 & 0.808 & 0.808 & 0.651 & 0.651 & 0.479 & 0.479 \\
\hline 7 & 15 & $1,318,834$ & 700,531 & 1 & 20,000 & Med. & 0.932 & 0.808 & 0.808 & 0.651 & 0.479 & 0.479 & 0.991 & 0.877 \\
\hline 7 & 30 & $1,318,834$ & 700,531 & 1 & 20,000 & Med. & 0.932 & 0.808 & 0.651 & 0.479 & 1 & 0.991 & 0.982 & 1 \\
\hline 7 & 60 & $1,318,834$ & 700,531 & 1 & 20,000 & Med. & 0.932 & 0.808 & 0.651 & 0.479 & 0.991 & 0.982 & 1 & 0.987 \\
\hline 8 & 1 & $1,235,072$ & 658,557 & 1 & 20,000 & Med. & 0.928 & 0.936 & 0.936 & 0.827 & 0.827 & 0.827 & 0.67 & 0.67 \\
\hline 8 & 5 & $1,235,072$ & 658,557 & 1 & 20,000 & Med. & 0.936 & 0.936 & 0.827 & 0.827 & 0.67 & 0.67 & 0.497 & 0.497 \\
\hline 8 & 15 & $1,235,072$ & 658,557 & 1 & 20,000 & Med. & 0.936 & 0.827 & 0.827 & 0.67 & 0.497 & 0.497 & 0.976 & 0.905 \\
\hline 8 & 30 & $1,235,072$ & 658,557 & 1 & 20,000 & Med. & 0.936 & 0.827 & 0.67 & 0.497 & 1 & 0.976 & 0.985 & 1 \\
\hline 8 & 60 & $1,235,072$ & 658,557 & 1 & 20,000 & Med. & 0.936 & 0.827 & 0.67 & 0.497 & 0.976 & 0.985 & 1 & 0.976 \\
\hline 9 & 1 & $1,146,524$ & 614,159 & 1 & 20,000 & Med. & 0.951 & 0.951 & 0.951 & 0.828 & 0.828 & 0.828 & 0.678 & 0.678 \\
\hline 9 & 5 & $1,146,524$ & 614,159 & 1 & 20,000 & Med. & 0.951 & 0.951 & 0.828 & 0.828 & 0.678 & 0.678 & 0.514 & 0.514 \\
\hline 9 & 15 & $1,146,524$ & 614,159 & 1 & 20,000 & Med. & 0.951 & 0.828 & 0.828 & 0.678 & 0.514 & 0.514 & 0.989 & 0.922 \\
\hline 9 & 30 & $1,146,524$ & 614,159 & 1 & 20,000 & Med. & 0.951 & 0.828 & 0.678 & 0.514 & 1 & 0.989 & 0.983 & 1 \\
\hline 9 & 60 & $1,146,524$ & 614,159 & 1 & 20,000 & Med. & 0.951 & 0.828 & 0.678 & 0.514 & 0.989 & 0.983 & 1 & 0.975 \\
\hline 10 & 1 & $1,337,332$ & 709,802 & 1 & 20,000 & Med. & 0.938 & 0.938 & 0.938 & 0.82 & 0.82 & 0.82 & 0.667 & 0.667 \\
\hline 10 & 5 & $1,337,332$ & 709,802 & 1 & 20,000 & Med. & 0.938 & 0.938 & 0.82 & 0.82 & 0.667 & 0.667 & 0.499 & 0.499 \\
\hline 10 & 15 & $1,337,332$ & 709,802 & 1 & 20,000 & Med. & 0.938 & 0.82 & 0.82 & 0.667 & 0.499 & 0.499 & 0.948 & 0.904 \\
\hline
\end{tabular}


Table S4 - Continued from previous page

\begin{tabular}{|c|c|c|c|c|c|c|c|c|c|c|c|c|c|c|}
\hline 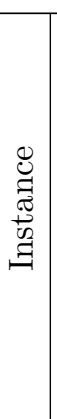 & 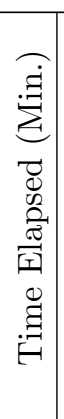 & $\begin{array}{l}\dot{\omega} \\
\dot{\vec{\omega}} \\
\dot{\nu}^{\circ} \\
\#\end{array}$ & $\begin{array}{l}\dot{a} \\
\stackrel{0}{0} \\
\text { \# }\end{array}$ & 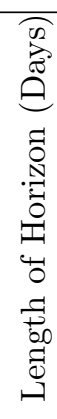 & 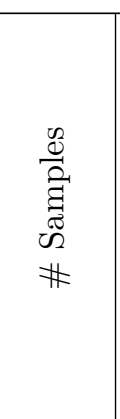 & $\stackrel{\mathbb{N}}{i n}$ & $\begin{array}{l}\stackrel{8}{\circ} \\
\stackrel{2}{Z} \\
z\end{array}$ & $\stackrel{8}{8}$ & $\frac{\stackrel{\curvearrowright}{\oplus}}{\stackrel{\rho}{\sigma}}$ & $\begin{array}{l}\stackrel{ }{H} \\
\stackrel{N}{S} \\
\stackrel{S}{S}\end{array}$ & $\begin{array}{l}8 \\
\stackrel{0}{0} \\
\text { S. } \\
1 \\
1 \\
1 \\
1\end{array}$ & 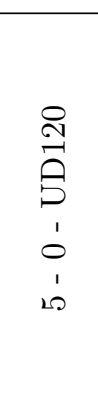 & $\begin{array}{l}\stackrel{0}{H} \\
\text { N } \\
\text { S } \\
1 \\
0 \\
1 \\
1\end{array}$ & 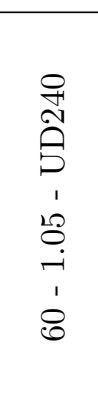 \\
\hline 10 & 30 & $1,337,332$ & 709,802 & 1 & 20,000 & Med. & 0.938 & 0.82 & 0.667 & 0.499 & 1 & 0.948 & 0.987 & 1 \\
\hline 10 & 60 & $1,337,332$ & 709,802 & 1 & 20,000 & Med. & 0.938 & 0.82 & 0.667 & 0.499 & 0.948 & 0.987 & 1 & 0.993 \\
\hline 11 & 1 & $1,973,660$ & $1,030,570$ & 1 & 30,000 & Med. & 0.941 & 0.941 & 0.941 & 0.78 & 0.78 & 0.78 & 0.649 & 0.649 \\
\hline 11 & 5 & $1,973,660$ & $1,030,570$ & 1 & 30,000 & Med. & 0.941 & 0.941 & 0.78 & 0.78 & 0.649 & 0.649 & 0.5 & 0.5 \\
\hline 11 & 15 & $1,973,660$ & $1,030,570$ & 1 & 30,000 & Med. & 0.941 & 0.78 & 0.78 & 0.649 & 0.5 & 0.5 & 0.985 & 0.761 \\
\hline 11 & 30 & $1,973,660$ & $1,030,570$ & 1 & 30,000 & Med. & 0.941 & 0.78 & 0.649 & 0.5 & 1 & 0.985 & 0.97 & 0.998 \\
\hline 11 & 60 & $1,973,660$ & $1,030,570$ & 1 & 30,000 & Med. & 0.941 & 0.78 & 0.649 & 0.5 & 0.985 & 0.97 & 1 & 0.975 \\
\hline 12 & 1 & $1,897,032$ & 992,192 & 1 & 30,000 & Med. & 0.934 & 0.934 & 0.934 & 0.799 & 0.799 & 0.799 & 0.661 & 0.661 \\
\hline 12 & 5 & $1,897,032$ & 992,192 & 1 & 30,000 & Med. & 0.934 & 0.934 & 0.799 & 0.799 & 0.661 & 0.661 & 0.509 & 0.509 \\
\hline 12 & 15 & $1,897,032$ & 992,192 & 1 & 30,000 & Med. & 0.934 & 0.799 & 0.799 & 0.661 & 0.509 & 0.509 & 0.975 & 0.767 \\
\hline 12 & 30 & $1,897,032$ & 992,192 & 1 & 30,000 & Med. & 0.934 & 0.799 & 0.661 & 0.509 & 2 & 0.975 & 0.964 & 1 \\
\hline 12 & 60 & $1,897,032$ & 992,192 & 1 & 30,000 & Med. & 0.934 & 0.799 & 0.661 & 0.509 & 0.975 & 0.964 & 1 & 0.985 \\
\hline 13 & 1 & $1,856,322$ & 971,578 & 1 & 30,000 & Med. & 0.932 & 0.932 & 0.932 & 0.791 & 0.791 & 0.791 & 0.667 & 0.667 \\
\hline 13 & 5 & $1,856,322$ & 971,578 & 1 & 30,000 & Med. & 0.932 & 0.932 & 0.791 & 0.791 & 0.667 & 0.667 & 0.516 & 0.516 \\
\hline 13 & 15 & $1,856,322$ & 971,578 & 1 & 30,000 & Med. & 0.932 & 0.791 & 0.791 & 0.667 & 0.516 & 0.516 & 0.938 & 0.667 \\
\hline 13 & 30 & $1,856,322$ & 971,578 & 1 & 30,000 & Med. & 0.932 & 0.791 & 0.667 & 0.516 & 1 & 0.938 & 0.962 & 1 \\
\hline 13 & 60 & $1,856,322$ & 971,578 & 1 & 30,000 & Med. & 0.932 & 0.791 & 0.667 & 0.516 & 0.938 & 0.962 & 1 & 0.966 \\
\hline 14 & 1 & $1,887,126$ & 987,090 & 1 & 30,000 & Med. & 0.936 & 0.936 & 0.936 & 0.782 & 0.782 & 0.782 & 0.651 & 0.651 \\
\hline 14 & 5 & $1,887,126$ & 987,090 & 1 & 30,000 & Med. & 0.936 & 0.936 & 0.782 & 0.782 & 0.651 & 0.651 & 0.501 & 0.501 \\
\hline 14 & 15 & $1,887,126$ & 987,090 & 1 & 30,000 & Med. & 0.936 & 0.782 & 0.782 & 0.651 & 0.501 & 0.501 & 0.967 & 0.881 \\
\hline 14 & 30 & $1,887,126$ & 987,090 & 1 & 30,000 & Med. & 0.936 & 0.782 & 0.651 & 0.501 & 1 & 0.967 & 0.97 & 1 \\
\hline 14 & 60 & $1,887,126$ & 987,090 & 1 & 30,000 & Med. & 0.936 & 0.782 & 0.651 & 0.501 & 0.967 & 0.97 & 1 & 0.969 \\
\hline 15 & 1 & $1,931,448$ & $1,009,384$ & 1 & 30,000 & Med. & 0.921 & 0.921 & 0.921 & 0.785 & 0.785 & 0.785 & 0.654 & 0.654 \\
\hline 15 & 5 & $1,931,448$ & $1,009,384$ & 1 & 30,000 & Med. & 0.921 & 0.921 & 0.785 & 0.785 & 0.654 & 0.654 & 0.503 & 0.503 \\
\hline
\end{tabular}


Table S4 - Continued from previous page

\begin{tabular}{|c|c|c|c|c|c|c|c|c|c|c|c|c|c|c|}
\hline 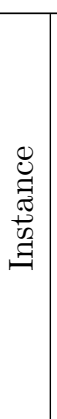 & 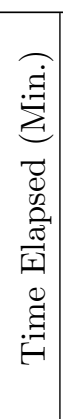 & $\begin{array}{l}\dot{\omega} \\
\dot{\vec{\omega}} \\
\dot{\nu}^{\circ} \\
\#\end{array}$ & $\begin{array}{l}\dot{a} \\
\stackrel{0}{0} \\
\text { \# }\end{array}$ & 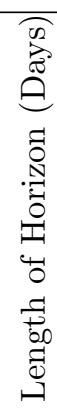 & 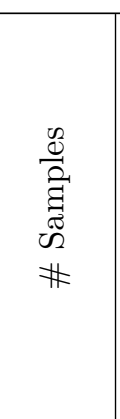 & $\stackrel{\mathbb{N}}{i n}$ & $\begin{array}{l}8 \\
\text { 号 } \\
\text { 乙 }\end{array}$ & $\stackrel{8}{8}$ & $\frac{\stackrel{\curvearrowright}{\oplus}}{\stackrel{\rho}{\sigma}}$ & $\begin{array}{l}\stackrel{ }{H} \\
\stackrel{N}{S} \\
\stackrel{S}{S}\end{array}$ & $\begin{array}{l}8 \\
\stackrel{0}{0} \\
\text { S. } \\
1 \\
1 \\
1 \\
1\end{array}$ & 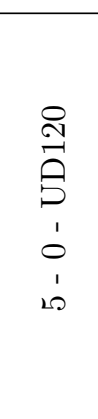 & $\begin{array}{l}\stackrel{0}{H} \\
\text { N } \\
\text { S } \\
1 \\
0 \\
1 \\
1\end{array}$ & 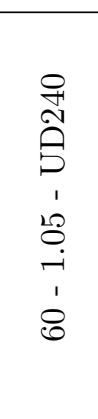 \\
\hline 15 & 15 & $1,931,448$ & $1,009,384$ & 1 & 30,000 & Med. & 0.921 & 0.785 & 0.785 & 0.654 & 0.503 & 0.503 & 0.953 & 0.865 \\
\hline 15 & 30 & $1,931,448$ & $1,009,384$ & 1 & 30,000 & Med. & 0.921 & 0.785 & 0.654 & 0.503 & 1 & 0.953 & 0.96 & 0.998 \\
\hline 15 & 60 & $1,931,448$ & $1,009,384$ & 1 & 30,000 & Med. & 0.921 & 0.785 & 0.654 & 0.503 & 0.953 & 0.96 & 1 & 0.964 \\
\hline 16 & 1 & $1,267,144$ & 707,713 & 2 & 10,000 & Med. & 0 & 1 & 1 & 0 & 0.868 & 0.868 & 0.743 & 0.743 \\
\hline 16 & 5 & $1,267,144$ & 707,713 & 2 & 10,000 & Med. & 0.987 & 1 & 0.868 & 0.868 & 0.743 & 0.743 & 0.515 & 0.515 \\
\hline 16 & 15 & $1,267,144$ & 707,713 & 2 & 10,000 & Med. & 1 & 0 & 0.868 & 0.743 & 0.515 & 0.515 & 0.989 & 0.743 \\
\hline 16 & 30 & $1,267,144$ & 707,713 & 2 & 10,000 & Med. & 1 & 0.868 & 0.743 & 0.515 & 2 & 0.989 & 0.977 & 0.96 \\
\hline 16 & 60 & $1,267,144$ & 707,713 & 2 & 10,000 & Med. & 1 & 0.868 & 0.743 & 0.515 & 0.989 & 0.977 & 0.96 & 0.963 \\
\hline 17 & 1 & $1,268,814$ & 708,545 & 2 & 10,000 & Med. & 0 & 1 & 1 & 0 & 0.866 & 0.866 & 0.733 & 0.733 \\
\hline 17 & 5 & $1,268,814$ & 708,545 & 2 & 10,000 & Med. & 0.989 & 1 & 0.866 & 0.866 & 0.733 & 0.733 & 0.515 & 0.515 \\
\hline 17 & 15 & $1,268,814$ & 708,545 & 2 & 10,000 & Med. & 1 & 0 & 0.866 & 0.733 & 0.515 & 0.515 & 0.982 & 0.764 \\
\hline 17 & 30 & $1,268,814$ & 708,545 & 2 & 10,000 & Med. & 1 & 0.866 & 0.733 & 0.515 & 2 & 0.982 & 0.977 & 0.981 \\
\hline 17 & 60 & $1,268,814$ & 708,545 & 2 & 10,000 & Med. & 1 & 0.866 & 0.733 & 0.515 & 0.982 & 0.977 & 0.981 & 0.951 \\
\hline 18 & 1 & $1,347,948$ & 748,069 & 2 & 10,000 & Med. & 0 & 0.992 & 0.992 & 0 & 0.828 & 0.828 & 0.718 & 0.718 \\
\hline 18 & 5 & $1,347,948$ & 748,069 & 2 & 10,000 & Med. & 0.979 & 0.992 & 0.828 & 0.828 & 0.718 & 0.718 & 0.508 & 0.508 \\
\hline 18 & 15 & $1,347,948$ & 748,069 & 2 & 10,000 & Med. & 0.992 & 0 & 0.828 & 0.718 & 0.508 & 0.508 & 0.985 & 0.95 \\
\hline 18 & 30 & $1,347,948$ & 748,069 & 2 & 10,000 & Med. & 0.992 & 0.828 & 0.718 & 0.508 & 2 & 1 & 0.973 & 0.965 \\
\hline 18 & 60 & $1,347,948$ & 748,069 & 2 & 10,000 & Med. & 0.992 & 0.828 & 0.718 & 0.508 & 1 & 0.973 & 0.965 & 0.916 \\
\hline 19 & 1 & $1,360,774$ & 754,589 & 2 & 10,000 & Med. & 0 & 1 & 1 & 0.845 & 0.854 & 0.854 & 0.714 & 0.714 \\
\hline 19 & 5 & $1,360,774$ & 754,589 & 2 & 10,000 & Med. & 0.989 & 1 & 0.854 & 0.854 & 0.714 & 0.714 & 0.516 & 0.516 \\
\hline 19 & 15 & $1,360,774$ & 754,589 & 2 & 10,000 & Med. & 1 & 0.845 & 0.854 & 0.714 & 0.516 & 0.516 & 0.988 & 0.898 \\
\hline 19 & 30 & $1,360,774$ & 754,589 & 2 & 10,000 & Med. & 1 & 0.854 & 0.714 & 0.516 & 2 & 0.988 & 0.932 & 0.967 \\
\hline 19 & 60 & $1,360,774$ & 754,589 & 2 & 10,000 & Med. & 1 & 0.854 & 0.714 & 0.516 & 0.988 & 0.932 & 0.967 & 0.948 \\
\hline 20 & 1 & $1,259,862$ & 704,040 & 2 & 10,000 & Med. & 0 & 0.993 & 0.993 & 0 & 0.869 & 0.869 & 0.764 & 0.764 \\
\hline
\end{tabular}


Table S4 - Continued from previous page

\begin{tabular}{|c|c|c|c|c|c|c|c|c|c|c|c|c|c|c|}
\hline 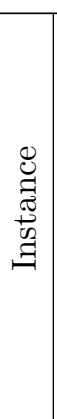 & 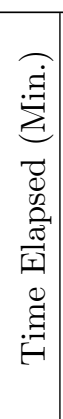 & $\begin{array}{l}\dot{\omega} \\
\dot{\vec{\omega}} \\
\dot{\nu}^{\circ} \\
\#\end{array}$ & $\begin{array}{l}\dot{a} \\
\stackrel{0}{0} \\
\text { \# } \\
\text { \# }\end{array}$ & 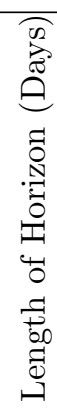 & 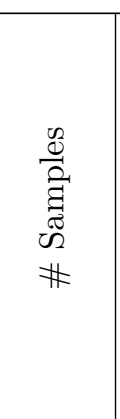 & $\stackrel{\mathbb{N}}{i n}$ & $\begin{array}{l}\stackrel{8}{\circ} \\
\stackrel{2}{Z} \\
z\end{array}$ & $\stackrel{8}{8}$ & $\frac{\stackrel{\curvearrowright}{\oplus}}{\stackrel{\rho}{\sigma}}$ & $\begin{array}{l}\stackrel{ }{H} \\
\stackrel{N}{S} \\
\stackrel{S}{S}\end{array}$ & $\begin{array}{l}8 \\
\mathscr{0} \\
S^{\prime} \\
1 \\
1 \\
1\end{array}$ & 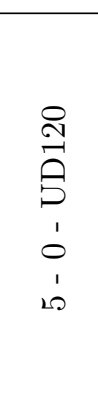 & $\begin{array}{l}\stackrel{0}{H} \\
\text { N } \\
\text { S } \\
1 \\
0 \\
1 \\
1\end{array}$ & 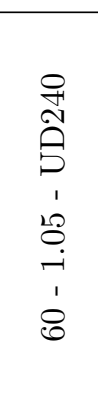 \\
\hline 20 & 5 & $1,259,862$ & 704,040 & 2 & 10,000 & Med. & 0.993 & 0.993 & 0.869 & 0.869 & 0.764 & 0.764 & 0.51 & 0.51 \\
\hline 20 & 15 & $1,259,862$ & 704,040 & 2 & 10,000 & Med. & 0.993 & 0 & 0.869 & 0.764 & 0.51 & 0.51 & 0.987 & 0.764 \\
\hline 20 & 30 & $1,259,862$ & 704,040 & 2 & 10,000 & Med. & 0.993 & 0.869 & 0.764 & 0.51 & 3 & 0.987 & 0.961 & 0.961 \\
\hline 20 & 60 & $1,259,862$ & 704,040 & 2 & 10,000 & Med. & 0.993 & 0.869 & 0.764 & 0.51 & 0.987 & 0.961 & 1 & 0.961 \\
\hline 21 & 1 & $1,910,338$ & $1,030,553$ & 2 & 15,000 & Med. & 0 & 1 & 1 & 0 & 0.863 & 0.863 & 0 & 0.719 \\
\hline 21 & 5 & $1,910,338$ & $1,030,553$ & 2 & 15,000 & Med. & 0 & 1 & 0 & 0.863 & 0.719 & 0.719 & 0.496 & 0.496 \\
\hline 21 & 15 & $1,910,338$ & $1,030,553$ & 2 & 15,000 & Med. & 1 & 0 & 0.863 & 0.719 & 0.496 & 0.496 & 0.995 & 0.719 \\
\hline 21 & 30 & $1,910,338$ & $1,030,553$ & 2 & 15,000 & Med. & 1 & 0.863 & 0.719 & 0.496 & 11 & 0.995 & 0.954 & 0.932 \\
\hline 21 & 60 & $1,910,338$ & $1,030,553$ & 2 & 15,000 & Med. & 1 & 0.863 & 0.719 & 0.496 & 0.995 & 0.954 & 0.932 & 0.937 \\
\hline 22 & 1 & $1,834,310$ & 992,558 & 2 & 15,000 & Med. & 0 & 1 & 1 & 0 & 0.9 & 0.9 & 0.726 & 0.726 \\
\hline 22 & 5 & $1,834,310$ & 992,558 & 2 & 15,000 & Med. & 0 & 1 & 0.898 & 0.9 & 0.726 & 0.726 & 0.511 & 0.511 \\
\hline 22 & 15 & $1,834,310$ & 992,558 & 2 & 15,000 & Med. & 1 & 0 & 0.9 & 0.726 & 0.511 & 0.511 & 0.992 & 0.726 \\
\hline 22 & 30 & $1,834,310$ & 992,558 & 2 & 15,000 & Med. & 1 & 0.9 & 0.726 & 0.511 & 9 & 0.992 & 0.96 & 0.93 \\
\hline 22 & 60 & $1,834,310$ & 992,558 & 2 & 15,000 & Med. & 1 & 0.9 & 0.726 & 0.511 & 0.992 & 0.96 & 0.98 & 0.897 \\
\hline 23 & 1 & $1,949,002$ & $1,049,765$ & 2 & 15,000 & Med. & 0 & 0.996 & 0.996 & 0 & 0.831 & 0.831 & 0.685 & 0.685 \\
\hline 23 & 5 & $1,949,002$ & $1,049,765$ & 2 & 15,000 & Med. & 0 & 0.996 & 0 & 0.831 & 0.685 & 0.685 & 0.48 & 0.48 \\
\hline 23 & 15 & $1,949,002$ & $1,049,765$ & 2 & 15,000 & Med. & 0.996 & 0 & 0.831 & 0.685 & 0.48 & 0.48 & 0.986 & 0.685 \\
\hline 23 & 30 & $1,949,002$ & $1,049,765$ & 2 & 15,000 & Med. & 0.996 & 0.831 & 0.685 & 0.48 & 4 & 0.986 & 1 & 0.975 \\
\hline 23 & 60 & $1,949,002$ & $1,049,765$ & 2 & 15,000 & Med. & 0.996 & 0.831 & 0.685 & 0.48 & 0.986 & 1 & 0.975 & 0.998 \\
\hline 24 & 1 & $1,892,194$ & $1,021,458$ & 2 & 15,000 & Med. & 0 & 0 & 1 & 0 & 0.875 & 0.875 & 0 & 0.7 \\
\hline 24 & 5 & $1,892,194$ & $1,021,458$ & 2 & 15,000 & Med. & 0 & 1 & 0 & 0.875 & 0.7 & 0.7 & 0.481 & 0.481 \\
\hline 24 & 15 & $1,892,194$ & $1,021,458$ & 2 & 15,000 & Med. & 0 & 0 & 0.875 & 0.7 & 0.481 & 0.481 & 0.868 & 0.7 \\
\hline 24 & 30 & $1,892,194$ & $1,021,458$ & 2 & 15,000 & Med. & 0.998 & 0.875 & 0.7 & 0.481 & 10 & 0.99 & 0.963 & 0.951 \\
\hline 24 & 60 & $1,892,194$ & $1,021,458$ & 2 & 15,000 & Med. & 1 & 0.875 & 0.7 & 0.481 & 0.99 & 0.963 & 0.978 & 0.881 \\
\hline
\end{tabular}


Table S4 - Continued from previous page

\begin{tabular}{|c|c|c|c|c|c|c|c|c|c|c|c|c|c|c|}
\hline 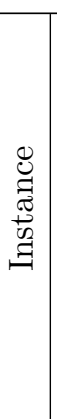 & 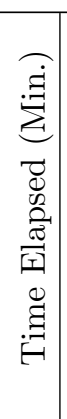 & $\begin{array}{l}\dot{\omega} \\
\dot{\vec{\omega}} \\
\dot{\nu}^{\circ} \\
\#\end{array}$ & $\begin{array}{l}\dot{a} \\
\stackrel{0}{0} \\
\text { \# } \\
\text { \# }\end{array}$ & 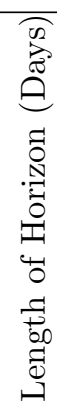 & 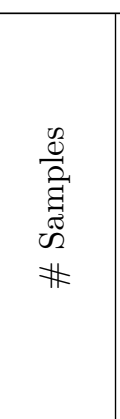 & $\stackrel{\mathbb{N}}{i n}$ & $\begin{array}{l}8 \\
\text { 号 } \\
\text { 乙 }\end{array}$ & $\stackrel{8}{8}$ & $\frac{\stackrel{\curvearrowright}{\oplus}}{\stackrel{\rho}{\sigma}}$ & $\begin{array}{l}\stackrel{ }{H} \\
\stackrel{N}{S} \\
\stackrel{S}{S}\end{array}$ & $\begin{array}{l}8 \\
\mathscr{0} \\
S^{\prime} \\
1 \\
1 \\
1\end{array}$ & 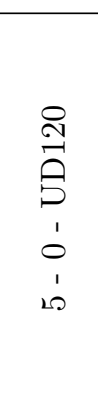 & $\begin{array}{l}\stackrel{0}{H} \\
\text { N } \\
\text { S } \\
1 \\
0 \\
1 \\
1\end{array}$ & 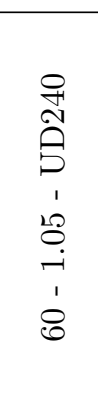 \\
\hline 25 & 1 & $1,740,642$ & 945,620 & 2 & 15,000 & Med. & 0 & 0.992 & 0.992 & 0 & 0.87 & 0.87 & 0.708 & 0.708 \\
\hline 25 & 5 & $1,740,642$ & 945,620 & 2 & 15,000 & Med. & 0 & 0.992 & 0 & 0.87 & 0.708 & 0.708 & 0.498 & 0.498 \\
\hline 25 & 15 & $1,740,642$ & 945,620 & 2 & 15,000 & Med. & 0.992 & 0 & 0.87 & 0.708 & 0.498 & 0.498 & 0.989 & 0.708 \\
\hline 25 & 30 & $1,740,642$ & 945,620 & 2 & 15,000 & Med. & 0.992 & 0.87 & 0.708 & 0.498 & 11 & 1 & 0.953 & 0.909 \\
\hline 25 & 60 & $1,740,642$ & 945,620 & 2 & 15,000 & Med. & 0.992 & 0.87 & 0.708 & 0.498 & 1 & 0.953 & 0.909 & 0.91 \\
\hline 26 & 1 & $1,955,878$ & $1,087,585$ & 3 & 10,000 & Med. & 0 & 0.944 & 1 & 0 & 0.828 & 0.828 & 0.011 & 0.794 \\
\hline 26 & 5 & $1,955,878$ & $1,087,585$ & 3 & 10,000 & Med. & 0 & 1 & 0 & 0.828 & 0.794 & 0.794 & 0.574 & 0.574 \\
\hline 26 & 15 & $1,955,878$ & $1,087,585$ & 3 & 10,000 & Med. & 0.944 & 0 & 0.828 & 0.794 & 0.574 & 0.574 & 0.958 & 0.011 \\
\hline 26 & 30 & $1,955,878$ & $1,087,585$ & 3 & 10,000 & Med. & 0.994 & 0.828 & 0.794 & 0.574 & 13 & 0.958 & 0.956 & 0.791 \\
\hline 26 & 60 & $1,955,878$ & $1,087,585$ & 3 & 10,000 & Med. & 1 & 0.828 & 0.794 & 0.574 & 0.958 & 0.956 & 0.791 & 0.896 \\
\hline 27 & 1 & $1,974,954$ & $1,097,106$ & 3 & 10,000 & Med. & 0 & 1 & 1 & 0 & 0.83 & 0.83 & 0 & 0.795 \\
\hline 27 & 5 & $1,974,954$ & $1,097,106$ & 3 & 10,000 & Med. & 0 & 1 & 0.83 & 0.83 & 0.795 & 0.795 & 0.61 & 0.61 \\
\hline 27 & 15 & $1,974,954$ & $1,097,106$ & 3 & 10,000 & Med. & 1 & 0 & 0.83 & 0.795 & 0.61 & 0.61 & 0.982 & 0 \\
\hline 27 & 30 & $1,974,954$ & $1,097,106$ & 3 & 10,000 & Med. & 1 & 0.83 & 0.795 & 0.61 & 21 & 0.982 & 0.957 & 0.959 \\
\hline 27 & 60 & $1,974,954$ & $1,097,106$ & 3 & 10,000 & Med. & 1 & 0.83 & 0.795 & 0.61 & 0.982 & 0.957 & 0.959 & 0.948 \\
\hline 28 & 1 & $1,997,392$ & $1,108,319$ & 3 & 10,000 & Med. & 0 & 0 & 1 & 0 & 0.812 & 0.812 & 0.775 & 0.775 \\
\hline 28 & 5 & $1,997,392$ & $1,108,319$ & 3 & 10,000 & Med. & 0 & 1 & 0.812 & 0.812 & 0.775 & 0.775 & 0.615 & 0.615 \\
\hline 28 & 15 & $1,997,392$ & $1,108,319$ & 3 & 10,000 & Med. & 0 & 0 & 0.812 & 0.775 & 0.615 & 0.615 & 0.982 & 0.775 \\
\hline 28 & 30 & $1,997,392$ & $1,108,319$ & 3 & 10,000 & Med. & 1 & 0.812 & 0.775 & 0.615 & 9 & 0.982 & 0.922 & 0.82 \\
\hline 28 & 60 & $1,997,392$ & $1,108,319$ & 3 & 10,000 & Med. & 1 & 0.812 & 0.775 & 0.615 & 0.982 & 0.922 & 0.82 & 0.942 \\
\hline 29 & 1 & $1,908,742$ & $1,063,943$ & 3 & 10,000 & Med. & 0 & 0.976 & 1 & 0 & 0.84 & 0.84 & 0 & 0.807 \\
\hline 29 & 5 & $1,908,742$ & $1,063,943$ & 3 & 10,000 & Med. & 0 & 1 & 0.83 & 0.84 & 0.807 & 0.807 & 0.632 & 0.632 \\
\hline 29 & 15 & $1,908,742$ & $1,063,943$ & 3 & 10,000 & Med. & 0.976 & 0 & 0.84 & 0.807 & 0.632 & 0.632 & 0.968 & 0 \\
\hline 29 & 30 & $1,908,742$ & $1,063,943$ & 3 & 10,000 & Med. & 1 & 0.84 & 0.807 & 0.632 & 11 & 0.968 & 0.976 & 0.947 \\
\hline
\end{tabular}


Table S4 - Continued from previous page

\begin{tabular}{|c|c|c|c|c|c|c|c|c|c|c|c|c|c|c|}
\hline 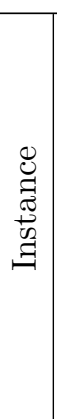 & 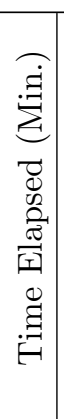 & $\begin{array}{l}\dot{\omega} \\
\dot{\vec{\omega}} \\
\dot{\nu}^{\circ} \\
\#\end{array}$ & $\begin{array}{l}\dot{a} \\
\stackrel{0}{0} \\
\text { \# }\end{array}$ & 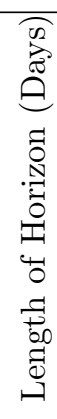 & 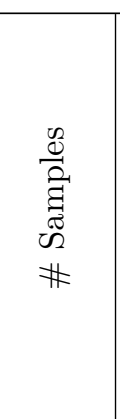 & $\stackrel{\mathbb{N}}{i n}$ & $\begin{array}{l}8 \\
\text { 号 } \\
\text { 乙 }\end{array}$ & $\stackrel{8}{8}$ & $\frac{\stackrel{\curvearrowright}{\oplus}}{\stackrel{\rho}{\sigma}}$ & $\begin{array}{l}\stackrel{ }{\sim} \\
\stackrel{\sim}{S}\end{array}$ & $\begin{array}{l}8 \\
\stackrel{0}{0} \\
\text { S. } \\
1 \\
1 \\
1 \\
1\end{array}$ & 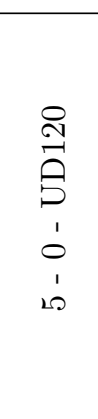 & $\begin{array}{l}\stackrel{0}{H} \\
\text { N } \\
\text { S } \\
1 \\
0 \\
1 \\
1\end{array}$ & 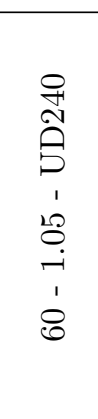 \\
\hline 29 & 60 & $1,908,742$ & $1,063,943$ & 3 & 10,000 & Med. & 1 & 0.84 & 0.807 & 0.632 & 0.968 & 0.976 & 0.947 & 0.886 \\
\hline 30 & 1 & $2,016,628$ & $1,117,913$ & 3 & 10,000 & Large & 0 & 0.974 & 1 & 0 & 0.822 & 0.822 & 0.01 & 0.784 \\
\hline 30 & 5 & $2,016,628$ & $1,117,913$ & 3 & 10,000 & Large & 0 & 1 & 0.822 & 0.822 & 0.784 & 0.784 & 0.585 & 0.585 \\
\hline 30 & 15 & $2,016,628$ & $1,117,913$ & 3 & 10,000 & Large & 0.974 & 0 & 0.822 & 0.784 & 0.585 & 0.585 & 0.987 & 0.01 \\
\hline 30 & 30 & $2,016,628$ & $1,117,913$ & 3 & 10,000 & Large & 1 & 0.822 & 0.784 & 0.585 & 8 & 0.987 & 0.899 & 0.942 \\
\hline 30 & 60 & $2,016,628$ & $1,117,913$ & 3 & 10,000 & Large & 1 & 0.822 & 0.784 & 0.585 & 0.987 & 0.899 & 0.942 & 0.951 \\
\hline 31 & 1 & 304,444 & 189,749 & 3 & 5,000 & Small & 0.997 & 0.997 & 0.997 & 0.941 & 0.941 & 0.941 & 0.889 & 0.889 \\
\hline 31 & 5 & 304,444 & 189,749 & 3 & 5,000 & Small & 0.997 & 0.997 & 0.941 & 0.941 & 0.889 & 0.889 & 0.61 & 0.61 \\
\hline 31 & 15 & 304,444 & 189,749 & 3 & 5,000 & Small & 0.997 & 0.941 & 0.941 & 0.889 & 0.61 & 0.61 & 0.997 & 0.998 \\
\hline 31 & 30 & 304,444 & 189,749 & 3 & 5,000 & Small & 0.997 & 0.941 & 0.889 & 0.61 & 0 & 0.997 & 0.998 & 0.997 \\
\hline 31 & 60 & 304,444 & 189,749 & 3 & 5,000 & Small & 0.997 & 0.941 & 0.889 & 0.61 & 0.997 & 0.998 & 0.997 & 1 \\
\hline 32 & 1 & 353,656 & 214,302 & 3 & 5,000 & Small & 0.999 & 0.999 & 0.999 & 0.943 & 0.943 & 0.943 & 0.892 & 0.892 \\
\hline 32 & 5 & 353,656 & 214,302 & 3 & 5,000 & Small & 0.999 & 0.999 & 0.943 & 0.943 & 0.892 & 0.892 & 0.581 & 0.581 \\
\hline 32 & 15 & 353,656 & 214,302 & 3 & 5,000 & Small & 0.999 & 0.943 & 0.943 & 0.892 & 0.581 & 0.581 & 0.999 & 1 \\
\hline 32 & 30 & 353,656 & 214,302 & 3 & 5,000 & Small & 0.999 & 0.943 & 0.892 & 0.581 & 0 & 0.999 & 1 & 0.999 \\
\hline 32 & 60 & 353,656 & 214,302 & 3 & 5,000 & Small & 0.999 & 0.943 & 0.892 & 0.581 & 0.999 & 1 & 0.999 & 0.999 \\
\hline 33 & 1 & 364,180 & 219,584 & 3 & 5,000 & Small & 0.998 & 0.998 & 0.998 & 0.872 & 0.872 & 0.872 & 0.824 & 0.824 \\
\hline 33 & 5 & 364,180 & 219,584 & 3 & 5,000 & Small & 0.998 & 0.998 & 0.872 & 0.872 & 0.824 & 0.824 & 0.584 & 0.584 \\
\hline 33 & 15 & 364,180 & 219,584 & 3 & 5,000 & Small & 0.998 & 0.872 & 0.872 & 0.824 & 0.584 & 0.584 & 0.997 & 0.993 \\
\hline 33 & 30 & 364,180 & 219,584 & 3 & 5,000 & Small & 0.998 & 0.872 & 0.824 & 0.584 & 0 & 0.997 & 1 & 0.997 \\
\hline 33 & 60 & 364,180 & 219,584 & 3 & 5,000 & Small & 0.998 & 0.872 & 0.824 & 0.584 & 0.997 & 1 & 0.997 & 1 \\
\hline 34 & 1 & 364,596 & 219,762 & 3 & 5,000 & Small & 1 & 1 & 1 & 0.932 & 0.932 & 0.932 & 0.887 & 0.887 \\
\hline 34 & 5 & 364,596 & 219,762 & 3 & 5,000 & Small & 1 & 1 & 0.932 & 0.932 & 0.887 & 0.887 & 0.608 & 0.608 \\
\hline 34 & 15 & 364,596 & 219,762 & 3 & 5,000 & Small & 1 & 0.932 & 0.932 & 0.887 & 0.608 & 0.608 & 0.999 & 0.997 \\
\hline
\end{tabular}


Table S4 - Continued from previous page

\begin{tabular}{|c|c|c|c|c|c|c|c|c|c|c|c|c|c|c|}
\hline 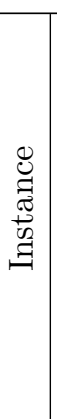 & 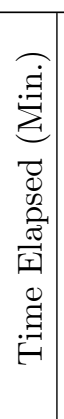 & $\begin{array}{l}\dot{\omega} \\
\dot{\vec{\omega}} \\
\dot{\nu}^{\circ} \\
\#\end{array}$ & $\begin{array}{l}\dot{a} \\
\stackrel{0}{0} \\
\text { \# } \\
\text { \# }\end{array}$ & 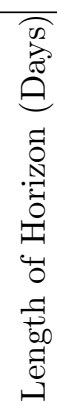 & 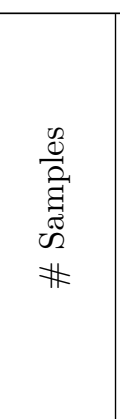 & $\stackrel{\mathbb{N}}{i n}$ & $\begin{array}{l}8 \\
\text { 号 } \\
\text { 乙 }\end{array}$ & 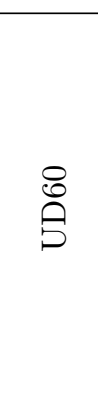 & $\frac{\stackrel{\curvearrowright}{\oplus}}{\stackrel{\rho}{\sigma}}$ & $\begin{array}{l}\stackrel{ }{\sim} \\
\stackrel{\sim}{S}\end{array}$ & $\begin{array}{l}8 \\
\stackrel{0}{0} \\
\vdots \\
1 \\
1 \\
1 \\
1\end{array}$ & 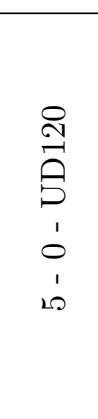 & $\begin{array}{l}\stackrel{ }{1} \\
\text { N } \\
\text { ! } \\
1 \\
0 \\
1 \\
1\end{array}$ & 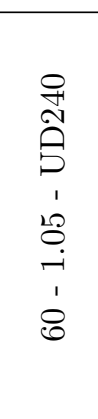 \\
\hline 34 & 30 & 364,596 & 219,762 & 3 & 5,000 & Small & 1 & 0.932 & 0.887 & 0.608 & 0 & 0.999 & 0.997 & 0.996 \\
\hline 34 & 60 & 364,596 & 219,762 & 3 & 5,000 & Small & 1 & 0.932 & 0.887 & 0.608 & 0.999 & 0.997 & 0.996 & 0.997 \\
\hline 35 & 1 & 344,038 & 209,449 & 3 & 5,000 & Small & 0.998 & 0.998 & 0.998 & 0.903 & 0.903 & 0.903 & 0.854 & 0.854 \\
\hline 35 & 5 & 344,038 & 209,449 & 3 & 5,000 & Small & 0.998 & 0.998 & 0.903 & 0.903 & 0.854 & 0.854 & 0.612 & 0.612 \\
\hline 35 & 15 & 344,038 & 209,449 & 3 & 5,000 & Small & 0.998 & 0.903 & 0.903 & 0.854 & 0.612 & 0.612 & 1 & 0.999 \\
\hline 35 & 30 & 344,038 & 209,449 & 3 & 5,000 & Small & 0.998 & 0.903 & 0.854 & 0.612 & 0 & 1 & 0.999 & 1 \\
\hline 35 & 60 & 344,038 & 209,449 & 3 & 5,000 & Small & 0.998 & 0.903 & 0.854 & 0.612 & 1 & 0.999 & 1 & 0.998 \\
\hline 36 & 1 & $2,709,636$ & $1,500,018$ & 4 & 10,000 & Large & 0 & 0 & 1 & 0 & 0.857 & 0.857 & 0 & 0.827 \\
\hline 36 & 5 & $2,709,636$ & $1,500,018$ & 4 & 10,000 & Large & 0 & 1 & 0.857 & 0.857 & 0.827 & 0.827 & 0.744 & 0.744 \\
\hline 36 & 15 & $2,709,636$ & $1,500,018$ & 4 & 10,000 & Large & 0 & 0 & 0.857 & 0.827 & 0.744 & 0.744 & 0.997 & 0 \\
\hline 36 & 30 & $2,709,636$ & $1,500,018$ & 4 & 10,000 & Large & 0.911 & 0.857 & 0.827 & 0.744 & 40 & 0.997 & 0.965 & 0.721 \\
\hline 36 & 60 & $2,709,636$ & $1,500,018$ & 4 & 10,000 & Large & 1 & 0.857 & 0.827 & 0.744 & 0.997 & 0.965 & 0.727 & 0.984 \\
\hline 37 & 1 & $2,276,894$ & $1,283,585$ & 4 & 10,000 & Large & 0 & 0 & 1 & 0 & 0.88 & 0.88 & 0 & 0.85 \\
\hline 37 & 5 & $2,276,894$ & $1,283,585$ & 4 & 10,000 & Large & 0 & 1 & 0 & 0.88 & 0.85 & 0.85 & 0.74 & 0.74 \\
\hline 37 & 15 & $2,276,894$ & $1,283,585$ & 4 & 10,000 & Large & 0 & 0 & 0.88 & 0.85 & 0 & 0.74 & 0.999 & 0 \\
\hline 37 & 30 & $2,276,894$ & $1,283,585$ & 4 & 10,000 & Large & 1 & 0.88 & 0.85 & 0 & 102 & 0.999 & 0.995 & 0.976 \\
\hline 37 & 60 & $2,276,894$ & $1,283,585$ & 4 & 10,000 & Large & 1 & 0.88 & 0.85 & 0.74 & 0.999 & 0.995 & 0.991 & 0.976 \\
\hline 38 & 1 & $2,760,022$ & $1,525,133$ & 4 & 10,000 & Large & 0 & 0 & 1 & 0 & 0.805 & 0.805 & 0.775 & 0.775 \\
\hline 38 & 5 & $2,760,022$ & $1,525,133$ & 4 & 10,000 & Large & 0 & 1 & 0.801 & 0.805 & 0.775 & 0.775 & 0.737 & 0.737 \\
\hline 38 & 15 & $2,760,022$ & $1,525,133$ & 4 & 10,000 & Large & 0 & 0 & 0.805 & 0.775 & 0.737 & 0.737 & 0.98 & 0.775 \\
\hline 38 & 30 & $2,760,022$ & $1,525,133$ & 4 & 10,000 & Large & 0.97 & 0.805 & 0.775 & 0.737 & 20 & 0.98 & 0.893 & 0.983 \\
\hline 38 & 60 & $2,760,022$ & $1,525,133$ & 4 & 10,000 & Large & 1 & 0.805 & 0.775 & 0.737 & 0.98 & 0.893 & 0.983 & 0.983 \\
\hline 39 & 1 & $2,438,780$ & $1,364,424$ & 4 & 10,000 & Large & 0 & 0.962 & 1 & 0 & 0.856 & 0.856 & 0 & 0.826 \\
\hline 39 & 5 & $2,438,780$ & $1,364,424$ & 4 & 10,000 & Large & 0 & 1 & 0.856 & 0.856 & 0.826 & 0.826 & 0.763 & 0.763 \\
\hline
\end{tabular}


Table S4 - Continued from previous page

\begin{tabular}{|c|c|c|c|c|c|c|c|c|c|c|c|c|c|c|}
\hline 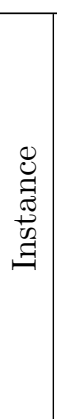 & 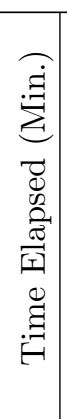 & $\begin{array}{l}\dot{\omega} \\
\dot{\vec{\omega}} \\
\dot{\nu}^{\circ} \\
\#\end{array}$ & $\begin{array}{l}\dot{a} \\
\stackrel{0}{0} \\
\text { \# }\end{array}$ & 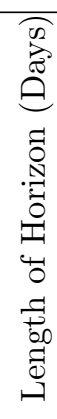 & 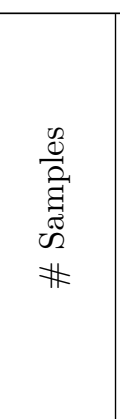 & $\stackrel{\mathbb{N}}{i s}$ & 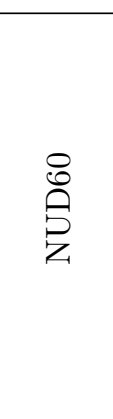 & $\stackrel{8}{8}$ & $\frac{\stackrel{\curvearrowright}{\oplus}}{\stackrel{\rho}{\sigma}}$ & $\begin{array}{l}\stackrel{ }{H} \\
\stackrel{N}{S} \\
\stackrel{S}{S}\end{array}$ & $\begin{array}{l}8 \\
\mathscr{0} \\
S^{\prime} \\
1 \\
1 \\
1\end{array}$ & 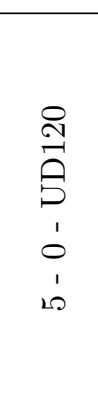 & $\begin{array}{l}\stackrel{0}{H} \\
\text { N } \\
\text { S } \\
1 \\
0 \\
1 \\
1\end{array}$ & 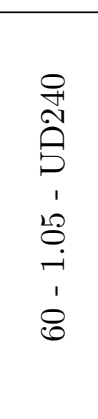 \\
\hline 39 & 15 & $2,438,780$ & $1,364,424$ & 4 & 10,000 & Large & 0.962 & 0 & 0.856 & 0.826 & 0.763 & 0.763 & 0.998 & 0.826 \\
\hline 39 & 30 & $2,438,780$ & $1,364,424$ & 4 & 10,000 & Large & 1 & 0.856 & 0.826 & 0.763 & 34 & 0.998 & 0.988 & 0.972 \\
\hline 39 & 60 & $2,438,780$ & $1,364,424$ & 4 & 10,000 & Large & 1 & 0.856 & 0.826 & 0.763 & 0.998 & 0.988 & 0.972 & 0.972 \\
\hline 40 & 1 & $2,537,444$ & $1,413,707$ & 4 & 10,000 & Large & 0 & 0 & 1 & 0 & 0.832 & 0.832 & 0.804 & 0.804 \\
\hline 40 & 5 & $2,537,444$ & $1,413,707$ & 4 & 10,000 & Large & 0 & 1 & 0.832 & 0.832 & 0.804 & 0.804 & 0.736 & 0.736 \\
\hline 40 & 15 & $2,537,444$ & $1,413,707$ & 4 & 10,000 & Large & 0 & 0 & 0.832 & 0.804 & 0.736 & 0.736 & 0.976 & 0.804 \\
\hline 40 & 30 & $2,537,444$ & $1,413,707$ & 4 & 10,000 & Large & 0.963 & 0.832 & 0.804 & 0.736 & 20 & 0.976 & 0.972 & 0.985 \\
\hline 40 & 60 & $2,537,444$ & $1,413,707$ & 4 & 10,000 & Large & 1 & 0.832 & 0.804 & 0.736 & 0.976 & 0.972 & 0.99 & 0.987 \\
\hline 41 & 1 & $1,375,486$ & 831,659 & 4 & 5,000 & Med. & 0 & 1 & 1 & 0.881 & 0.881 & 0.881 & 0.835 & 0.835 \\
\hline 41 & 5 & $1,375,486$ & 831,659 & 4 & 5,000 & Med. & 1 & 1 & 0.881 & 0.881 & 0.835 & 0.835 & 0.806 & 0.806 \\
\hline 41 & 15 & $1,375,486$ & 831,659 & 4 & 5,000 & Med. & 1 & 0.881 & 0.881 & 0.835 & 0.806 & 0.806 & 0.998 & 0.983 \\
\hline 41 & 30 & $1,375,486$ & 831,659 & 4 & 5,000 & Med. & 1 & 0.881 & 0.835 & 0.806 & 6 & 0.998 & 0.999 & 0.999 \\
\hline 41 & 60 & $1,375,486$ & 831,659 & 4 & 5,000 & Med. & 1 & 0.881 & 0.835 & 0.806 & 0.998 & 0.999 & 0.999 & 1 \\
\hline 42 & 1 & $1,315,546$ & 801,605 & 4 & 5,000 & Med. & 0 & 1 & 1 & 0.882 & 0.882 & 0.882 & 0.84 & 0.84 \\
\hline 42 & 5 & $1,315,546$ & 801,605 & 4 & 5,000 & Med. & 1 & 1 & 0.882 & 0.882 & 0.84 & 0.84 & 0.814 & 0.814 \\
\hline 42 & 15 & $1,315,546$ & 801,605 & 4 & 5,000 & Med. & 1 & 0.882 & 0.882 & 0.84 & 0.814 & 0.814 & 0.998 & 0.995 \\
\hline 42 & 30 & $1,315,546$ & 801,605 & 4 & 5,000 & Med. & 1 & 0.882 & 0.84 & 0.814 & 4 & 0.998 & 1 & 1 \\
\hline 42 & 60 & $1,315,546$ & 801,605 & 4 & 5,000 & Med. & 1 & 0.882 & 0.84 & 0.814 & 0.998 & 1 & 1 & 1 \\
\hline 43 & 1 & $1,376,592$ & 832,173 & 4 & 5,000 & Med. & 0.988 & 1 & 1 & 0 & 0.862 & 0.862 & 0.813 & 0.813 \\
\hline 43 & 5 & $1,376,592$ & 832,173 & 4 & 5,000 & Med. & 1 & 1 & 0.862 & 0.862 & 0.813 & 0.813 & 0.778 & 0.778 \\
\hline 43 & 15 & $1,376,592$ & 832,173 & 4 & 5,000 & Med. & 1 & 0 & 0.862 & 0.813 & 0.778 & 0.778 & 0.999 & 0.951 \\
\hline 43 & 30 & $1,376,592$ & 832,173 & 4 & 5,000 & Med. & 1 & 0.862 & 0.813 & 0.778 & 8 & 0.999 & 0.999 & 1 \\
\hline 43 & 60 & $1,376,592$ & 832,173 & 4 & 5,000 & Med. & 1 & 0.862 & 0.813 & 0.778 & 0.999 & 0.999 & 1 & 1 \\
\hline 44 & 1 & $1,306,294$ & 797,005 & 4 & 5,000 & Med. & 0.998 & 0.998 & 0.998 & 0.88 & 0.88 & 0.88 & 0.835 & 0.835 \\
\hline
\end{tabular}


Table S4 - Continued from previous page

\begin{tabular}{|c|c|c|c|c|c|c|c|c|c|c|c|c|c|c|}
\hline 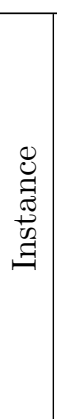 & 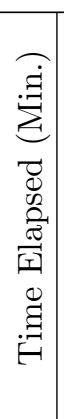 & $\begin{array}{l}\dot{\omega} \\
\dot{\vec{\omega}} \\
\dot{\nu}^{\circ} \\
\#\end{array}$ & $\begin{array}{l}\dot{a} \\
\stackrel{0}{0} \\
\text { \# } \\
\text { \# }\end{array}$ & 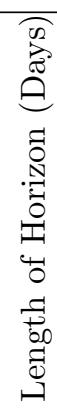 & 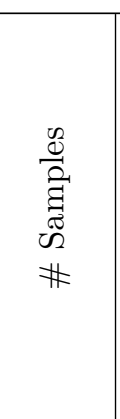 & $\stackrel{\mathbb{N}}{i n}$ & $\begin{array}{l}\stackrel{8}{\circ} \\
\stackrel{2}{Z} \\
z\end{array}$ & $\stackrel{8}{8}$ & $\frac{\stackrel{\curvearrowright}{\oplus}}{\stackrel{\rho}{\sigma}}$ & $\begin{array}{l}\stackrel{ }{H} \\
\stackrel{N}{S} \\
\stackrel{S}{S}\end{array}$ & $\begin{array}{l}8 \\
\mathscr{0} \\
S^{\prime} \\
1 \\
1 \\
1\end{array}$ & 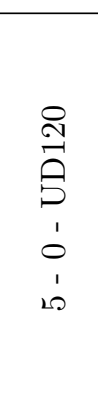 & $\begin{array}{l}\stackrel{0}{H} \\
\text { N } \\
\text { S } \\
1 \\
0 \\
1 \\
1\end{array}$ & 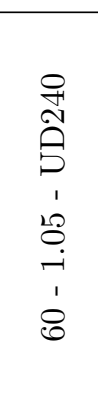 \\
\hline 44 & 5 & $1,306,294$ & 797,005 & 4 & 5,000 & Med. & 0.998 & 0.998 & 0.88 & 0.88 & 0.835 & 0.835 & 0.808 & 0.808 \\
\hline 44 & 15 & $1,306,294$ & 797,005 & 4 & 5,000 & Med. & 0.998 & 0.88 & 0.88 & 0.835 & 0.808 & 0.808 & 1 & 0.981 \\
\hline 44 & 30 & $1,306,294$ & 797,005 & 4 & 5,000 & Med. & 0.998 & 0.88 & 0.835 & 0.808 & 5 & 1 & 1 & 1 \\
\hline 44 & 60 & $1,306,294$ & 797,005 & 4 & 5,000 & Med. & 0.998 & 0.88 & 0.835 & 0.808 & 1 & 1 & 1 & 0.998 \\
\hline 45 & 1 & $1,252,562$ & 770,184 & 4 & 5,000 & Med. & 0 & 1 & 1 & 0 & 0.928 & 0.928 & 0.88 & 0.88 \\
\hline 45 & 5 & $1,252,562$ & 770,184 & 4 & 5,000 & Med. & 1 & 1 & 0.928 & 0.928 & 0.88 & 0.88 & 0.854 & 0.854 \\
\hline 45 & 15 & $1,252,562$ & 770,184 & 4 & 5,000 & Med. & 1 & 0 & 0.928 & 0.88 & 0.854 & 0.854 & 1 & 0.98 \\
\hline 45 & 30 & $1,252,562$ & 770,184 & 4 & 5,000 & Med. & 1 & 0.928 & 0.88 & 0.854 & 7 & 1 & 0.996 & 0.996 \\
\hline 45 & 60 & $1,252,562$ & 770,184 & 4 & 5,000 & Med. & 1 & 0.928 & 0.88 & 0.854 & 1 & 0.996 & 0.996 & 1 \\
\hline 46 & 1 & $3,305,890$ & $1,833,463$ & 5 & 10,000 & Large & 0 & 0 & 0.931 & 0 & 0.848 & 0.848 & 0.816 & 0.816 \\
\hline 46 & 5 & $3,305,890$ & $1,833,463$ & 5 & 10,000 & Large & 0 & 0.931 & 0 & 0.848 & 0.816 & 0.816 & 0.791 & 0.791 \\
\hline 46 & 15 & $3,305,890$ & $1,833,463$ & 5 & 10,000 & Large & 0 & 0 & 0.848 & 0.816 & 0.791 & 0.791 & 1 & 0.816 \\
\hline 46 & 30 & $3,305,890$ & $1,833,463$ & 5 & 10,000 & Large & 0 & 0.848 & 0.816 & 0.791 & 50 & 1 & 0.971 & 0.726 \\
\hline 46 & 60 & $3,305,890$ & $1,833,463$ & 5 & 10,000 & Large & 0.931 & 0.848 & 0.816 & 0.791 & 1 & 0.971 & 0.726 & 0.988 \\
\hline 47 & 1 & $3,234,804$ & $1,798,044$ & 5 & 10,000 & Large & 0 & 0 & 0.998 & 0 & 0.851 & 0.851 & 0 & 0.815 \\
\hline 47 & 5 & $3,234,804$ & $1,798,044$ & 5 & 10,000 & Large & 0 & 0.998 & 0 & 0.851 & 0.815 & 0.815 & 0.787 & 0.787 \\
\hline 47 & 15 & $3,234,804$ & $1,798,044$ & 5 & 10,000 & Large & 0 & 0 & 0.851 & 0.815 & 0.787 & 0.787 & 0.997 & 0 \\
\hline 47 & 30 & $3,234,804$ & $1,798,044$ & 5 & 10,000 & Large & 0.979 & 0.851 & 0.815 & 0.787 & 48 & 0.997 & 1 & 0.698 \\
\hline 47 & 60 & $3,234,804$ & $1,798,044$ & 5 & 10,000 & Large & 0.998 & 0.851 & 0.815 & 0.787 & 0.997 & 1 & 0.698 & 0.998 \\
\hline 48 & 1 & $3,399,906$ & $1,880,512$ & 5 & 10,000 & Large & 0 & 0 & 1 & 0 & 0.836 & 0.836 & 0.799 & 0.799 \\
\hline 48 & 5 & $3,399,906$ & $1,880,512$ & 5 & 10,000 & Large & 0 & 1 & 0.834 & 0.836 & 0.799 & 0.799 & 0.772 & 0.772 \\
\hline 48 & 15 & $3,399,906$ & $1,880,512$ & 5 & 10,000 & Large & 0 & 0 & 0.836 & 0.799 & 0.772 & 0.772 & 0.997 & 0.799 \\
\hline 48 & 30 & $3,399,906$ & $1,880,512$ & 5 & 10,000 & Large & 0 & 0.836 & 0.799 & 0.772 & 15 & 0.997 & 0.974 & 0.982 \\
\hline 48 & 60 & $3,399,906$ & $1,880,512$ & 5 & 10,000 & Large & 1 & 0.836 & 0.799 & 0.772 & 0.997 & 0.974 & 0.982 & 0.982 \\
\hline
\end{tabular}


Table S4 - Continued from previous page

\begin{tabular}{|c|c|c|c|c|c|c|c|c|c|c|c|c|c|c|}
\hline 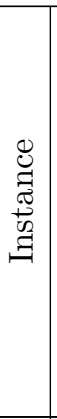 & 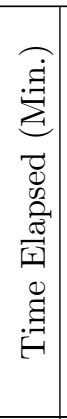 & 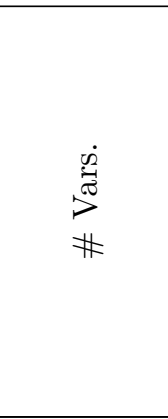 & $\begin{array}{l}\dot{\Xi} \\
\dot{0} \\
0 \\
\#\end{array}$ & 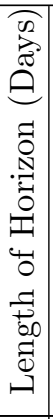 & 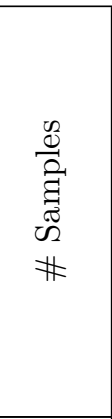 & 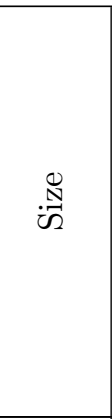 & $\frac{8}{\circ}$ & 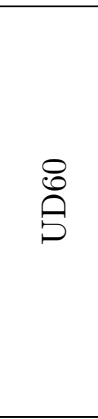 & 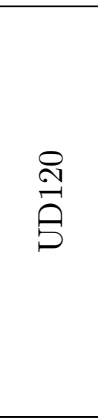 & 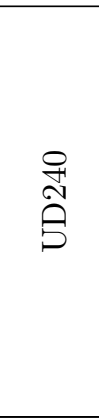 & $\begin{array}{l}8 \\
\stackrel{0}{0} \\
1 \\
1 \\
1 \\
1\end{array}$ & $\begin{array}{l}\stackrel{\Im}{N} \\
\vec{D} \\
\vdots \\
1 \\
0 \\
1 \\
10\end{array}$ & 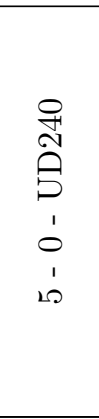 & 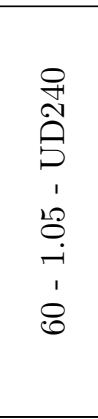 \\
\hline 49 & 1 & $3,192,728$ & $1,776,841$ & 5 & 10,000 & Large & 0 & 0 & 0.998 & 0 & 0.841 & 0.841 & 0 & 0.808 \\
\hline 49 & 5 & $3,192,728$ & $1,776,841$ & 5 & 10,000 & Large & 0 & 0.998 & 0 & 0.841 & 0.808 & 0.808 & 0.77 & 0.77 \\
\hline 49 & 15 & $3,192,728$ & $1,776,841$ & 5 & 10,000 & Large & 0 & 0 & 0.841 & 0.808 & 0.77 & 0.77 & 1 & 0.808 \\
\hline 49 & 30 & $3,192,728$ & $1,776,841$ & 5 & 10,000 & Large & 0.926 & 0.841 & 0.808 & 0.77 & 49 & 1 & 0.996 & 0.7 \\
\hline 49 & 60 & $3,192,728$ & $1,776,841$ & 5 & 10,000 & Large & 0.998 & 0.841 & 0.808 & 0.77 & 1 & 0.996 & 0.7 & 0.974 \\
\hline 50 & 1 & $3,339,016$ & $1,850,121$ & 5 & 10,000 & Large & 0 & 0 & 0.95 & 0 & 0.858 & 0.858 & 0 & 0.822 \\
\hline 50 & 5 & $3,339,016$ & $1,850,121$ & 5 & 10,000 & Large & 0 & 0.95 & 0 & 0.858 & 0.822 & 0.822 & 0.793 & 0.793 \\
\hline 50 & 15 & $3,339,016$ & $1,850,121$ & 5 & 10,000 & Large & 0 & 0 & 0.858 & 0.822 & 0.793 & 0.793 & 0.983 & 0 \\
\hline 50 & 30 & $3,339,016$ & $1,850,121$ & 5 & 10,000 & Large & 0 & 0.858 & 0.822 & 0.793 & 37 & 0.983 & 0.985 & 1 \\
\hline 50 & 60 & $3,339,016$ & $1,850,121$ & 5 & 10,000 & Large & 0.95 & 0.858 & 0.822 & 0.793 & 0.983 & 0.985 & 1 & 1 \\
\hline 51 & 1 & $1,743,006$ & $1,050,816$ & 5 & 5,000 & Med. & 0 & 1 & 1 & 0.879 & 0.879 & 0.879 & 0.815 & 0.815 \\
\hline 51 & 5 & $1,743,006$ & $1,050,816$ & 5 & 5,000 & Med. & 0 & 1 & 0.879 & 0.879 & 0.815 & 0.815 & 0.778 & 0.778 \\
\hline 51 & 15 & $1,743,006$ & $1,050,816$ & 5 & 5,000 & Med. & 1 & 0.879 & 0.879 & 0.815 & 0.778 & 0.778 & 0.995 & 0.994 \\
\hline 51 & 30 & $1,743,006$ & $1,050,816$ & 5 & 5,000 & Med. & 1 & 0.879 & 0.815 & 0.778 & 3 & 0.995 & 0.999 & 1 \\
\hline 51 & 60 & $1,743,006$ & $1,050,816$ & 5 & 5,000 & Med. & 1 & 0.879 & 0.815 & 0.778 & 0.995 & 0.999 & 1 & 0.992 \\
\hline 52 & 1 & $1,657,794$ & $1,008,232$ & 5 & 5,000 & Med. & 0 & 1 & 1 & 0 & 0.896 & 0.896 & 0.84 & 0.84 \\
\hline 52 & 5 & $1,657,794$ & $1,008,232$ & 5 & 5,000 & Med. & 1 & 1 & 0.896 & 0.896 & 0.84 & 0.84 & 0.809 & 0.809 \\
\hline 52 & 15 & $1,657,794$ & $1,008,232$ & 5 & 5,000 & Med. & 1 & 0 & 0.896 & 0.84 & 0.809 & 0.809 & 0.997 & 0.998 \\
\hline 52 & 30 & $1,657,794$ & $1,008,232$ & 5 & 5,000 & Med. & 1 & 0.896 & 0.84 & 0.809 & 3 & 0.997 & 0.998 & 0.99 \\
\hline 52 & 60 & $1,657,794$ & $1,008,232$ & 5 & 5,000 & Med. & 1 & 0.896 & 0.84 & 0.809 & 0.997 & 0.998 & 0.99 & 1 \\
\hline 53 & 1 & $1,832,988$ & $1,095,905$ & 5 & 5,000 & Med. & 0 & 0.996 & 0.996 & 0.908 & 0.908 & 0.908 & 0.85 & 0.85 \\
\hline 53 & 5 & $1,832,988$ & $1,095,905$ & 5 & 5,000 & Med. & 0 & 0.996 & 0.908 & 0.908 & 0.85 & 0.85 & 0.818 & 0.818 \\
\hline 53 & 15 & $1,832,988$ & $1,095,905$ & 5 & 5,000 & Med. & 0.996 & 0.908 & 0.908 & 0.85 & 0.818 & 0.818 & 1 & 0.982 \\
\hline 53 & 30 & $1,832,988$ & $1,095,905$ & 5 & 5,000 & Med. & 0.996 & 0.908 & 0.85 & 0.818 & 3 & 1 & 0.986 & 0.98 \\
\hline
\end{tabular}


Table S4 - Continued from previous page

\begin{tabular}{|c|c|c|c|c|c|c|c|c|c|c|c|c|c|c|}
\hline 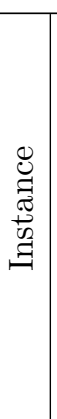 & 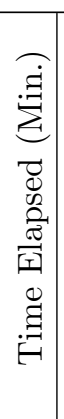 & $\begin{array}{l}\dot{\omega} \\
\dot{\vec{\omega}} \\
\dot{\nu}^{\circ} \\
\#\end{array}$ & $\begin{array}{l}\dot{a} \\
\stackrel{0}{0} \\
\text { \# }\end{array}$ & 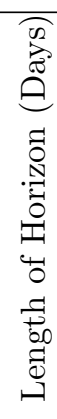 & 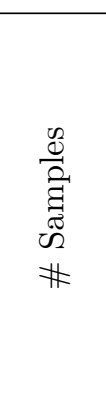 & $\stackrel{\mathbb{N}}{i n}$ & $\begin{array}{l}\stackrel{8}{\circ} \\
\stackrel{2}{Z} \\
z\end{array}$ & $\stackrel{8}{8}$ & $\frac{\stackrel{\curvearrowright}{\oplus}}{\stackrel{\rho}{\sigma}}$ & $\begin{array}{l}\stackrel{ }{H} \\
\stackrel{N}{S} \\
\stackrel{S}{S}\end{array}$ & $\begin{array}{l}8 \\
\mathscr{0} \\
S^{\prime} \\
1 \\
1 \\
1\end{array}$ & 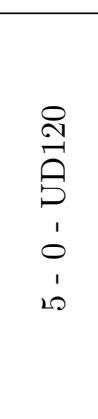 & $\begin{array}{l}\stackrel{0}{H} \\
\text { N } \\
\text { S } \\
1 \\
0 \\
1 \\
1\end{array}$ & 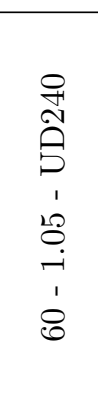 \\
\hline 53 & 60 & $1,832,988$ & $1,095,905$ & 5 & 5,000 & Med. & 0.996 & 0.908 & 0.85 & 0.818 & 1 & 0.986 & 0.98 & 0.979 \\
\hline 54 & 1 & $1,665,390$ & $1,012,064$ & 5 & 5,000 & Med. & 0 & 1 & 1 & 0 & 0.937 & 0.937 & 0.877 & 0.877 \\
\hline 54 & 5 & $1,665,390$ & $1,012,064$ & 5 & 5,000 & Med. & 1 & 1 & 0.937 & 0.937 & 0.877 & 0.877 & 0.841 & 0.841 \\
\hline 54 & 15 & $1,665,390$ & $1,012,064$ & 5 & 5,000 & Med. & 1 & 0 & 0.937 & 0.877 & 0.841 & 0.841 & 0.996 & 0.994 \\
\hline 54 & 30 & $1,665,390$ & $1,012,064$ & 5 & 5,000 & Med. & 1 & 0.937 & 0.877 & 0.841 & 4 & 0.996 & 1 & 0.991 \\
\hline 54 & 60 & $1,665,390$ & $1,012,064$ & 5 & 5,000 & Med. & 1 & 0.937 & 0.877 & 0.841 & 0.996 & 1 & 0.991 & 0.991 \\
\hline 55 & 1 & $1,734,194$ & $1,046,389$ & 5 & 5,000 & Med. & 0 & 0.999 & 0.999 & 0.889 & 0.889 & 0.889 & 0.833 & 0.833 \\
\hline 55 & 5 & $1,734,194$ & $1,046,389$ & 5 & 5,000 & Med. & 0.992 & 0.999 & 0.889 & 0.889 & 0.833 & 0.833 & 0.804 & 0.804 \\
\hline 55 & 15 & $1,734,194$ & $1,046,389$ & 5 & 5,000 & Med. & 0.999 & 0.889 & 0.889 & 0.833 & 0.804 & 0.804 & 0.999 & 1 \\
\hline 55 & 30 & $1,734,194$ & $1,046,389$ & 5 & 5,000 & Med. & 0.999 & 0.889 & 0.833 & 0.804 & 2 & 0.999 & 1 & 0.999 \\
\hline 55 & 60 & $1,734,194$ & $1,046,389$ & 5 & 5,000 & Med. & 0.999 & 0.889 & 0.833 & 0.804 & 0.999 & 1 & 0.999 & 0.999 \\
\hline 56 & 1 & $2,452,604$ & $1,476,603$ & 7 & 5,000 & Large & 0 & 0 & 0.998 & 0 & 0.901 & 0.901 & 0.833 & 0.833 \\
\hline 56 & 5 & $2,452,604$ & $1,476,603$ & 7 & 5,000 & Large & 0 & 0.998 & 0.901 & 0.901 & 0.833 & 0.833 & 0.794 & 0.794 \\
\hline 56 & 15 & $2,452,604$ & $1,476,603$ & 7 & 5,000 & Large & 0 & 0 & 0.901 & 0.833 & 0.794 & 0.794 & 1 & 0.833 \\
\hline 56 & 30 & $2,452,604$ & $1,476,603$ & 7 & 5,000 & Large & 0.998 & 0.901 & 0.833 & 0.794 & 7 & 1 & 0.996 & 0.998 \\
\hline 56 & 60 & $2,452,604$ & $1,476,603$ & 7 & 5,000 & Large & 0.998 & 0.901 & 0.833 & 0.794 & 1 & 0.996 & 0.998 & 0.998 \\
\hline 57 & 1 & $2,667,758$ & $1,584,269$ & 7 & 5,000 & Large & 0 & 0 & 1 & 0 & 0.955 & 0.955 & 0.877 & 0.877 \\
\hline 57 & 5 & $2,667,758$ & $1,584,269$ & 7 & 5,000 & Large & 0 & 1 & 0.955 & 0.955 & 0.877 & 0.877 & 0.835 & 0.835 \\
\hline 57 & 15 & $2,667,758$ & $1,584,269$ & 7 & 5,000 & Large & 0 & 0 & 0.955 & 0.877 & 0.835 & 0.835 & 0.995 & 0.877 \\
\hline 57 & 30 & $2,667,758$ & $1,584,269$ & 7 & 5,000 & Large & 1 & 0.955 & 0.877 & 0.835 & 7 & 0.995 & 0.994 & 1 \\
\hline 57 & 60 & $2,667,758$ & $1,584,269$ & 7 & 5,000 & Large & 1 & 0.955 & 0.877 & 0.835 & 0.995 & 0.994 & 1 & 1 \\
\hline 58 & 1 & $2,082,018$ & $1,291,228$ & 7 & 5,000 & Large & 0 & 1 & 1 & 0 & 0.977 & 0.977 & 0.901 & 0.901 \\
\hline 58 & 5 & $2,082,018$ & $1,291,228$ & 7 & 5,000 & Large & 0 & 1 & 0.977 & 0.977 & 0.901 & 0.901 & 0.864 & 0.864 \\
\hline 58 & 15 & $2,082,018$ & $1,291,228$ & 7 & 5,000 & Large & 1 & 0 & 0.977 & 0.901 & 0.864 & 0.864 & 0.998 & 1 \\
\hline
\end{tabular}


Table S4 - Continued from previous page

\begin{tabular}{|c|c|c|c|c|c|c|c|c|c|c|c|c|c|c|}
\hline 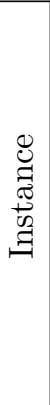 & 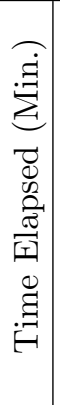 & 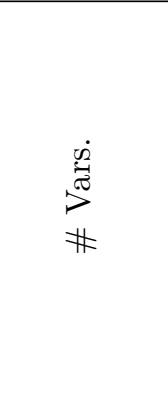 & $\begin{array}{l}\dot{\Xi} \\
\dot{0} \\
0 \\
\text { \# }\end{array}$ & 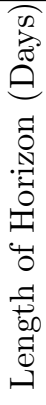 & 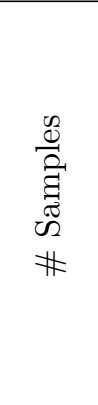 & $\stackrel{0}{N}$ & $\begin{array}{l}\stackrel{8}{\circ} \\
\text { 总 }\end{array}$ & 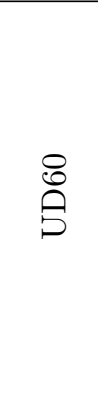 & $\stackrel{\stackrel{\sim}{0}}{\vec{\rho}}$ & $\begin{array}{l}\stackrel{H}{N} \\
\stackrel{\sim}{S} \\
\text { S }\end{array}$ & 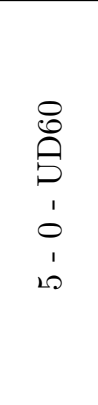 & $\begin{array}{l}\stackrel{0}{N} \\
\overrightarrow{\mid} \\
\vdots \\
1 \\
1 \\
10\end{array}$ & $\begin{array}{l}\stackrel{P}{H} \\
\stackrel{N}{1} \\
\text { S } \\
1 \\
0 \\
1 \\
1\end{array}$ & 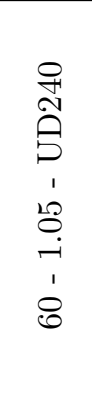 \\
\hline 58 & 30 & $2,082,018$ & $1,291,228$ & 7 & 5,000 & Large & 1 & 0.977 & 0.901 & 0.864 & 13 & 0.998 & 1 & 0.996 \\
\hline 58 & 60 & $2,082,018$ & $1,291,228$ & 7 & 5,000 & Large & 1 & 0.977 & 0.901 & 0.864 & 0.998 & 1 & 0.996 & 0.996 \\
\hline 59 & 1 & $2,078,980$ & $1,289,713$ & 7 & 5,000 & Large & 0 & 0.996 & 0.996 & 0 & 0.996 & 0.996 & 0 & 0.936 \\
\hline 59 & 5 & $2,078,980$ & $1,289,713$ & 7 & 5,000 & Large & 0 & 0.996 & 0 & 0.996 & 0.936 & 0.936 & 0.892 & 0.892 \\
\hline 59 & 15 & $2,078,980$ & $1,289,713$ & 7 & 5,000 & Large & 0.996 & 0 & 0.996 & 0.936 & 0.892 & 0.892 & 0.996 & 0.936 \\
\hline 59 & 30 & $2,078,980$ & $1,289,713$ & 7 & 5,000 & Large & 0.996 & 0.996 & 0.936 & 0.892 & 34 & 0.996 & 1 & 0.997 \\
\hline 59 & 60 & $2,078,980$ & $1,289,713$ & 7 & 5,000 & Large & 0.996 & 0.996 & 0.936 & 0.892 & 0.996 & 1 & 0.997 & 0.997 \\
\hline 60 & 1 & $2,515,326$ & $1,508,049$ & 7 & 5,000 & Large & 0 & 0 & 1 & 0 & 0.956 & 0.956 & 0 & 0.88 \\
\hline 60 & 5 & $2,515,326$ & $1,508,049$ & 7 & 5,000 & Large & 0 & 1 & 0 & 0.956 & 0.88 & 0.88 & 0.842 & 0.842 \\
\hline 60 & 15 & $2,515,326$ & $1,508,049$ & 7 & 5,000 & Large & 0 & 0 & 0.956 & 0.88 & 0.842 & 0.842 & 0.996 & 0.88 \\
\hline 60 & 30 & $2,515,326$ & $1,508,049$ & 7 & 5,000 & Large & 0.979 & 0.956 & 0.88 & 0.842 & 55 & 0.996 & 0.996 & 0.997 \\
\hline 60 & 60 & $2,515,326$ & $1,508,049$ & 7 & 5,000 & Large & 1 & 0.956 & 0.88 & 0.842 & 0.996 & 0.996 & 0.997 & 0.997 \\
\hline
\end{tabular}

